\title{
Fuel and diluent property effects during wet compression of a fuel aerosol under RCM conditions
}

S.S. Goldsborough ${ }^{\mathrm{a} *}$, M.V. Johnson ${ }^{\mathrm{b}}$, G.S. Zhu' ${ }^{\mathrm{c}}$, and S.K. Aggarwal ${ }^{\mathrm{d}}$

a Department of Mechanical Engineering

Marquette University

PO Box 1881

Milwaukee, WI 53201-1881

Ph: 414-288-6641; Fax: 414-288-7790

b Energy Systems Division

Argonne National Laboratory

9700 S. Cass Avenue

Argonne, IL 60439-4815

Ph: 630-252-3529; Fax: 630-252-3443

c HDEP Performance \& Emissions

DTNA -- Detroit Diesel Corporation

13400 Outer Dr. West

Detroit, MI 48239

Ph: 313-592 5896, Fax: 313-592 5906

d Department of Mechanical and Industrial Engineering

University of Illinois at Chicago

1030 Engineering Research Facility

842 W. Taylor Street

Chicago, IL 60607

Ph: 312-413-9712, Fax: 312-413-0447

*corresponding author

scott.goldsborough@mu.edu

Ph: 001-414-288-6641; Fax: 001-414-288-7790

This is a full-length article 


\begin{abstract}
Wet compression of fuel aerosols has been proposed as a means of creating gas-phase mixtures of involatile diesel-representative fuels and oxidizer + diluent gases for rapid compression machine (RCM) experiments. The intent of this study is to investigate the effects of fuel and diluent gas properties on the wet compression process, specifically to: (a) explore a range of fuels which could have applicability in aerosol RCM experiments, and (b) fundamentally understand how fuel and diluent gas properties affect the wet compression process and assess which ones are most important. Insight gained from this work can be utilized to aid the design and successful operation of aerosol RCMs. A spherically-symmetric, single-droplet wet compression model is used where $n$-Heptane, $n$-dodecane, 2,2,4,4,6,8,8heptamethylnonane (isocetane), $n$-hexadecane (cetane) and $n$-eicosane are investigated as the dieselrepresentative fuels, while comparisons are made to water droplets. Nitrogen, neon and argon are selected as the gas-phase diluents while the oxidizer is considered to be oxygen at atmospheric concentrations. Initial droplet diameters of $d_{0}=3$ and $8 \mu \mathrm{m}$ are used based on results of previous studies where the overall compression time is set to $15.3 \mathrm{~ms}$ with the maximum volumetric compression ratio
\end{abstract} 13.4. An overall equivalence ratio of $\varphi=1.0$ is used.

It is shown that under these conditions, involatile fuels up to $\sim n$-hexadecane appear to be candidates for aerosol RCM experiments. However, the use of small droplets $\left(d_{0}<5 \mu \mathrm{m}\right)$ will be necessary in order to ensure complete vaporization and adequate gas-phase mixing in advance of low temperature chemical reactivity. Fuels with higher boiling points might not be useable unless extremely small droplets $\left(d_{0}<\right.$ $1 \mu \mathrm{m})$ and low pressures (e.g., $P_{0}<0.5$ bar) are employed along with longer compression times. In addition, the boiling curve (i.e., saturation pressure) and $L_{f}$ are found to be the dominant fuel properties while the density-weighted mass diffusivity, $\rho_{g} D_{g}$, which controls the rate of gas phase mass diffusion, and thus compositional stratification, generally plays a secondary role. The heat capacity and molar mass are the dominant diluent properties that affect the near-droplet and 'far-field' conditions. The gas-phase mixture Lewis number $\left(L e_{g}\right)$ contributes to either greater compositional $\left(L e_{g}>1\right)$ or thermal $\left(L e_{g}<1\right)$ stratification. For large hydrocarbons and oxygenated hydrocarbons that are representative of diesel fuels $L e_{g} \sim 3-5$, and therefore compositional stratification could be significant; this characteristic has the potential to complicate interpretation of ignition/oxidation data acquired from these machines. 


\section{Key Words}

Wet compression

Droplet evaporation

Aerosol RCM

Transient model

Diesel-surrogate

\section{Nomenclature}

$\begin{array}{ll}\mathrm{c}_{\mathrm{p}} & \text { specific heat at constant pressure } \\ \mathrm{c}_{\mathrm{v}} & \text { specific heat at constant volume } \\ D & \text { mass diffusion coefficient } \\ d & \text { diameter } \\ K n & \text { Knudsen number }\left(\eta / r_{s}\right) \\ L & \text { heat of vaporization } \\ L e & \text { Lewis number ( } / D) \\ \dot{m} & \text { net evaporation rate } \\ \bar{m} & \text { non-dimensional evaporation rate, } \dot{m} / 4 \pi \rho_{g} D_{g} r_{s} \\ M & \text { molar mass } \\ P & \text { pressure } \\ r & \text { radial direction } \\ \bar{t} & \text { non-dimensional time } \int \hat{\kappa}_{l} d t / r_{s}^{2}\end{array}$




$\begin{array}{ll}T & \text { temperature } \\ u & \text { radial velocity } \\ Y & \text { mass fraction, vapor phase }\end{array}$

\section{Greeks}

$\begin{array}{ll}\alpha & \text { thermal conductivity } \\ \eta & \text { mean free path } \\ \varphi & \text { equivalence ratio } \\ \kappa & \text { heat diffusivity } \\ \rho & \text { density } \\ \phi & \text { fugacity coefficient }\end{array}$

$\begin{array}{ll}\text { Subscripts } & \\ \text { comp } & \text { 'air' } \\ f & \text { fuel } \\ g & \text { gas-phase } \\ k & k^{\text {th }} \text { species } \\ l & \text { liquid-phase } \\ s & \text { droplet surface } \\ \text { w } & \text { water } \\ 0 & \text { initial value } \\ \infty & \text { 'far-field' value }\end{array}$




\section{Introduction}

Wet compression is the process whereby vaporization is achieved via volumetric compression heating of the gas-phase of a droplet laden aerosol. This phenomenon has received increasing attention in the power generation field in recent years with applications to advanced combustion processes and alternative fuel formulations in internal combustion engines [1-7], and to continuous cooling configurations using water aerosols in gas turbine engines [8-10]. In shock tubes (STs) and rapid compression machines (RCMs) wet compression has also been proposed as a means of preparing test gases for high molecular weight, involatile liquid fuels relevant to the transportation industry. Fundamental ignition and intermediate speciation data for such fuels is extremely scarce, especially at engine-relevant conditions, but are necessary towards the development and design of advanced combustion engines and novel fuel formulations. Traditional charge preparation techniques for these laboratory apparatuses generally use external mixing protocols based on partial pressure methodologies $[11,12]$. Diesel-representative fuels however, have extremely low vapor pressures at standard conditions (e.g., $P^{\text {sat }}<1$ Torr) which make this option very difficult or impossible. Heating the mixing tanks and test apparatus can lead to better fuel vaporization but this is still limited in RCMs due to seal degradation issues, and there is concern for pretest chemical reactivity during the mixture preparation process. Aerosols of suspended fuel droplets $\left(d_{\text {mean }} \sim 8-18 \mu \mathrm{m}\right)$ have been used to deliver liquid fuels to a shock tube and an RCM using flow-through techniques (i.e., inlet and outlet valves located at opposite ends of the device), where subsequent volumetric compression of the gas-phase results in evaporation of the liquid fuel droplets [13-15]. In shock tubes where test temperatures generally range from 900 to $2000 \mathrm{~K}$ the gas-phase compression event is achieved via a rapidly traveling shock wave $\left(\Delta t_{\text {comp }} \sim 30 \mu \mathrm{s} ; T_{\text {comp }} \sim 600-700 \mathrm{~K}\right)$; the passing of the initial compression wave not only increases the pressure and temperature of the surrounding bath gases but it fragments the initial droplets and results in high convective velocities near the droplet surface. These features result in very fast vaporization (e.g., $\Delta t_{\text {evap }} \sim 100 \mu$ s [13]). The subsequent reflected wave compresses the evaporated fuel + oxidizer + diluent mixture to the test conditions. In RCMs, where test 
temperatures generally range from 600 to $1100 \mathrm{~K}$, the compression event is much slower (e.g., $\Delta t_{\text {comp }} \sim 15$ $60 \mathrm{~ms}$ ) and bulk gas fluid dynamics (i.e., on the scale of the reaction chamber) are often suppressed through the use of machined piston crevices in order to minimize unwanted heat loss during the test period [16]. As a result, droplet evaporation in aerosol RCMs will therefore be much slower and generally constrained by the initial droplet size and ensuing gas-phase diffusion processes. Evaporation must also be achieved at lower temperatures than in STs (e.g., by $\sim 500 \mathrm{~K}$ to precede low temperature chemical reactivity).

The design and operation of aerosol RCMs which can effectively utilize the wet compression process for involatile diesel-representative fuels requires a fundamental understanding of droplet evaporation including both near-droplet and 'far-field' processes (i.e., due to droplet-droplet interactions). It is very important to also assess the limitations of the wet compression process and gauge to what extent homogeneous gas-phase mixtures can be created for various fuels and diluent gas mixtures. This study builds upon previous work where an integrated simulation tool was developed, and here we investigate important issues for aerosol RCM applications. The use of high aerosol concentrations (e.g., $\sim 0.1 \mathrm{~mL}_{\text {fuel }} / \mathrm{L}_{\text {gas }}, \sim 1 \times 10^{9}$ droplets per liter of gas), which are necessary to achieve desired fuel loadings for investigation of engine-relevant chemistry (e.g., $\varphi=0.5-2.0,5-21 \% \mathrm{O}_{2}, P_{\text {comp }}=10-50 \mathrm{bar}$ ), can result in droplet-droplet interactions which lead to substantial gas-phase fuel saturation and evaporative cooling during the piston compression process. These conditions can alter the rate of evaporation and the extent of volumetric compression required to achieve complete vaporization. In addition, localized stratification (i.e., on the droplet scale) of the fuel vapor and of temperature can occur due to insufficient rates of transport; this could lead to non-homogeneous chemical reaction and heat release - features which may prevent an adequate segregation of the chemical kinetic rates from rates of physical transport. These operational characteristics are dependent on many factors including fuel and diluent properties such as the boiling curve (i.e., saturation pressure), vaporization enthalpy, heat capacity, molar mass, and mass / 
thermal diffusivities, as well as physical parameters such as overall fuel loading and initial droplet size relative to the compression rate.

The objective of this study is to investigate fuel and diluent gas property effects in order to better understand dominant factors during wet compression. Specifically, we: (a) explore a range of fuels with potential applicability to aerosol RCM experiments and illustrate important limitations due to fuel properties, and (b) isolate gas phase properties to fundamentally understand how these affect the wet compression process, thereby determining which ones are most important. The results of this work can be used to aid the successful design and operation of aerosol RCMs. Previous studies by our group have investigated effects of physical parameters where $n$-dodecane, a relatively small involatile hydrocarbon, was used as the fuel $[17,18]$. In those studies it was shown that smaller droplets are more affected by gasphase fuel stratification and evaporative cooling while larger droplets result in greater gas-phase compositional and thermal stratification. These effects are due to the differences in diffusion distances across which the fuel must travel from, and the heat to the droplet. It was also demonstrated previously that shorter compression times can lead to greater gas-phase stratification due to the non-linear rate of temperature (and thus saturation pressure) rise, and the relatively slower rates of diffusion of fuel from and heat to the droplet. Lower gas pressures were found to enhance evaporation during wet compression due to the higher fuel mass fraction at the droplet surface, where this increases the rate of fuel transport across the gas phase.

In this work a spherically-symmetric, single-droplet wet compression model is employed where five hydrocarbons with a range boiling and transport characteristics are now considered including $n$-heptane, $n$-dodecane, 2,2,4,4,6,8,8-heptamethylnonane (HMN, or isocetane), $n$-hexadecane (cetane) and $n$ eicosane; comparisons are also made to the wet compression behavior of water aerosols. Three diluent gases including nitrogen, neon and argon are studied while oxygen mole fractions of $21 \%$ are used as the balance of the non-fuel gas. Initial droplet diameters of $d_{0}=3$ and $8 \mu \mathrm{m}$ are considered based on results of our previous studies [18]. These droplet sizes are much smaller than typically produced by fuel injectors, but could be created via an ultra-sonic nebulizing system or similar device (e.g., see ref. $[13,15]$ ). 
Compression times and extent of compression (i.e., maximum compression ratio, $C R_{\max }=V_{0} / V_{\min }$ )

representative of typical RCMs are used. The remainder of this paper is organized as follows. First, an overview of the wet compression model is presented along with a brief discussion of modifications undertaken for this study. Simulation results are then discussed covering the various liquids and diluent gas mixtures at the two droplet sizes. For the various liquids two cases are considered: one, where identical initial conditions are used in order to highlight boiling curve effects, and a second, where the initial temperatures are modified in order to highlight effects of other properties including $L_{f}$ and $\rho_{g} D_{g}$. Differences in the near-droplet and far-field behavior are discussed for these cases. Finally, issues concerning successful operation of an aerosol RCM are discussed.

\section{Model}

This study utilizes the wet compression model described previously by Goldsborough and co-workers $[17,18]$. The model integrates a fully transient evaporation sub-model developed by Aggarwal and coworkers [19-21] with a gas-phase volumetric compression sub-model. The evaporation sub-model has been extensively validated and employs a continuum formulation with phase and thermal equilibria prescribed at the liquid-vapor interface. This prescription can lead to errors in the predicted evaporation times for the droplets investigated here due to gas kinetic effects at small droplet diameters (e.g., $d_{0}$ $<5 \mu \mathrm{m})$. However, these errors are generally modest $(+5-15 \%)$ and the trends in the simulation results using the interface equilibria prescription are comparable to those observed with a refined modeling approach where gas kinetic effects are taken into account (e.g., see refs. [18,22-25]). The droplet and surrounding gas-phase are assumed to be spherically symmetric, and thermal and concentration gradients within the droplet are taken into account through the application of relevant conservation equations. Absorption of the oxidizer and diluent gas into the liquid phase is also considered through the use of the fugacity coefficient, however the effect of this here is negligible. In addition, the droplet sizes used in this study are small enough however so that no internal stratification is seen. The evaporation model is diffusion-limited, meaning that the droplet Reynolds number $\left(R e_{d}\right)$ is assumed to be zero. This is a 
reasonable approximation since it is expected that the fuel aerosol will be generated ex-situ to the machine and delivered to it using a flow-through technique (e.g., see refs. $[13,15])$. Device-scale Reynolds numbers (i.e., using the cylinder bore as the length scale) near 500 are expected to be used in order to prevent droplet settling and wall impingement [26]; this can be converted to $R e_{d}$ of 0.01 or less. While some residual fluid motion or turbulent behavior resulting from the aerosol delivery process would aid the mixing of the vapor phase fuel with the oxidizer + diluent, and improve heat transport to the droplets, the use of a diffusion-limited model provides a lower bound for the rates of these transport processes and the resulting localized (i.e., on the droplet scale) compositional and thermal stratification. Spatial variations in pressure are taken into account in the model, as are variations in the liquid- and gas-phase properties due to the large changes in temperature and pressure experienced under some conditions. For all of the cases explored in this study however, the pressure is found to be spatially uniform during evaporation. Real gas equations of state (e.g., Peng-Robinson (PR-EOS) [27], as is used in this work) are facilitated in the software, though the gas-phase compressibility factors $(z=P / \rho R T)$ for all of the cases investigated here are found to be nearly 1.0 so that an ideal gas model could be reasonably used instead. A mesh compression operation is integrated with the evaporation model in order to simulate the gas-phase compression heating process. This sub-model accounts for the induced radial flow towards the droplet due to volumetric compression. The evaporation sub-model has been detailed previously in refs. [19-21] where predictions are validated against a range of experimental data. In addition, this sub-model has been extensively validated for the transient evaporation of an $n$-heptane droplet under high pressure conditions [19]. Application of the integrated model to study wet compression of an isolated $n$-dodecane droplet (i.e., $\varphi \sim 0.0)$ has been described in refs. $[17,18]$. While the droplet evaporation model has been extensively validated, there is currently a lack of experimental data however for validation of the integrated wet compression model. Thus there is some uncertainty concerning the model performance. In ref. [17] other approaches for modeling wet compression have been reviewed and compared. It was demonstrated in that work that simplified evaporation models, such as a quasi-steady formulation, can 
yield significant discrepancies (e.g., $+50 \%$ in total evaporation time) compared to results predicted by a fully transient formulation, such as employed here.

The wet compression model is modified from ref. [17] in order to investigate fuel and diluent gas property effects near the droplet and at the 'far-field' under RCM conditions. Figure 1 illustrates the configuration implemented for this study. A single droplet is used to represent the fuel loaded into the $\mathrm{RCM}$ for an experiment while the gas-phase domain is constrained in order to represent the finite volume of the RCM apparatus. It is assumed that the aerosol is mono-dispersed and uniformly distributed throughout the reaction chamber. This approximation, while not quite representative of an externally generated aerosol (e.g., see ref. [13]), provides a useful approach for understanding the effects of gas phase saturation and evaporative cooling. It can also yield insights regarding limitations on maximum acceptable droplet size, for a range of fuels and diluent gases.

No-flux (i.e., no-gradient) boundary conditions are applied at the edge of the gas-phase domain (i.e., the 'far-field') to ensure that mass, momentum, energy and species cannot enter or leave the reaction chamber volume (i.e., $\partial \rho_{k, \infty} / \partial r=0, \partial \rho_{\infty} / \partial r=0, u_{\infty}=0$, and $\partial T_{\infty} / \partial r=0$ at $r=r_{\infty}$ ). The heat loss from the reaction chamber mixture is thus not taken into account in the current model. While this can substantially influence the thermal and concentration homogeneity, as well as fuel evaporation during aerosol RCM experiments, it is a device-scale phenomenon and thus not appropriate to investigate using this single droplet model. A more suitable framework for studying this is a multi-zone model that is currently under development by the primary author (e.g., see ref. [28]).

The overall fuel-air ratio for the simulation is specified according to the equivalence ratio definition $\varphi=\frac{\left(m_{f} / m_{a}\right)}{\left(m_{f} / m_{a}\right)_{\text {stoich }}}=\frac{1}{0.067} \frac{\rho_{f, 0} r_{s, 0}^{3}}{\rho_{a, 0}\left(r_{\infty, 0}^{3}-r_{s, 0}^{3}\right)}$

where $m_{f}$ and $m_{a}$ refer to the mass of fuel and 'air' (or oxygen + diluent), respectively. The subscript 'stoich' refers to the stoichiometric condition while the subscript ' $O$ ' indicates the initial conditions for the simulation. The value of 0.067 in this expression is the stoichiometric fuel-air ratio for $n$-dodecane $\left(\left(m_{f}\right.\right.$ $\left.\left(m_{a}\right)_{\text {stoich }}\right)$; stoichiometric ratios for the other hydrocarbons are provided in Table 1. The initial densities of 
the fuel and 'air', $\rho_{f, 0}$ and $\rho_{a, 0}$, respectively, are dependent on the initial temperature and pressure used in a particular experiment; once these are specified along with the initial droplet radius, $r_{s, 0}$, the initial 'farfield' boundary used in the simulation, $r_{\infty, 0}$, can be located. Table 1 lists geometric boundaries for the six liquids investigated in this study where an overall equivalence ratio of $\varphi=1.0$ is prescribed; the initial temperature and pressure conditions are discussed in the next section. It can be seen here that larger droplet sizes require greater 'far-field' distances for the same fuel loading, while larger values of $\varphi$ require smaller gas volumes for the same droplet size.

The time-varying location of the 'far-field' boundary which governs the rate of compression heating is dependent on the volumetric history of the RCM. This is expressed using the time-varying compression ratio

$$
C R(t)=\frac{V_{0}}{V(t)}=\frac{r_{\infty, 0}^{3}}{r_{\infty}^{3}(t)}
$$

For this work we have used $C R(t)$ as an input table where the time history is representative of the operational characteristics of RCMs currently utilized for chemical kinetic studies [16,29-30].

More details of the wet compression model, including the governing equations and issues associated with thermodynamic non-equilibrium at the droplet surface can be found in ref. [18]. A summary of the thermo-physical models important to this study is presented next. The gas- and liquid-phase thermophysical properties are considered to be functions of pressure, temperature and composition with the activity coefficient equal to 1 (i.e., the ideal solution model). The method suggested by Chung et al. [31] is employed to calculate the viscosity of the gas mixture while the thermal conductivity is based on the group contribution model of Roy and Thodos [32-33]. The binary gas-phase mass diffusivity is calculated using the empirical model of Fuller et al. [34] with corrections for pressure effects using the Takahashi correlation [35]. For multi-component mixtures, the effective diffusivity is obtained using the formula given by Bird et al. [36]. The gas density is calculated directly from the PR-EOS as is the liquidvapor equilibrium condition, including $\phi_{k}$ and $L_{k}$, which are the fugacity coefficient and vaporization enthalpy of species $k$, respectively. The enthalpy of the gas mixture is based on a molar weighting of the 
pure component enthalpies obtained from the CHEMKIN database [37] (computed using the THERM software [38]). A generalized thermodynamic correlation based on a three-parameter corresponding states method [39] is used to compute the enthalpy correction for high-pressure effects. For the conditions explored in this study however, and those relevant to typical RCM experiments, $z \sim 1$ so that simplified thermo-physical models could reasonably be employed in analogous simulations, or data reduction calculations.

The liquid-phase properties are determined using two approaches. For the five hydrocarbons the following is used. The heat capacity of pure components is calculated based on the group contribution model described by Rùzicka and Domalski [40] and then extended to mixtures (for the absorbed oxygen + diluent gases) using Filippov's rule [41]. The thermal conductivity and mass diffusivity are obtained using the correlations of Latini et al. [34] and Nakanishi [42], respectively. The density is calculated based on a polynomial fit of experimental data [43-47], with these recorded near 1atm, along with the high pressure correction given by Thomson et al. [48]. The viscosity is computed using a power law fit of experimental data near 1atm [46-47,49]. For water, the pure component heat capacity, thermal conductivity, density and viscosity are calculated using polynomial fits of experimental data near 1atm, with these extended to high pressures, and mixtures with the absorbed gases using methods described above.

In addition to the results presented in the next section, results for a sensitivity study of the wet compression model are discussed in the Appendix where normalized sensitivity coefficients are computed for various fuel and diluent gas properties. It is shown that for $n$-dodecane at the conditions of this study, $\rho_{l, f}, L_{f}, D_{g, f}, \phi_{f}^{l}, c_{p g, d i l}$, and $\alpha_{g, d i l}$ are the most sensitive parameters. This is consistent with the findings of the analyses presented in section 3.0, and provides insight into the phenomena influencing the wet compression process.

\section{Model Results}


The results presented in this section are for five hydrocarbons, four of which have been employed previously as single-species, or as constituents of multi-component diesel surrogates including $n$-heptane, $n$-dodecane, HMN and $n$-hexadecane [50]. A range of volatilities and other physical properties typical of diesel are covered with these surrogate fuels, a few of which are listed in Table 1. $n$-Eicosane is utilized in this study to represent some of the most involatile petro- or bio-diesel components where typical T90 distillation points are near $600 \mathrm{~K}$ for petro- and near $670 \mathrm{~K}$ for bio-diesel [51]. Only single-species fuel droplets are investigated in this work, though the model could also be utilized to study effects of multicomponent fuel mixes. To investigate real fuel behavior however, where many species are present including hundreds of isomers [50] and a range of boiling and transport properties exist, requires modifications to the model, as discussed in ref. [52]. Investigation of such complex blends is not often conducted in RCM experiments however where two to three component mixes are generally more typical. The investigation of preferential, or fractional distillation and gas-phase diffusion for multi-component fuel mixes, while of interest towards understanding and ensuring compositional homogeneity during aerosol RCM experiments, is beyond the scope of this study. Water is used in this study to illustrate the effects of significantly different boiling and transport characteristics on the wet compression process. Neon and argon are selected as diluents in addition to nitrogen. Argon is often used in RCM experiments due to its high specific heat ratio (i.e., $\mathrm{c}_{\mathrm{p}} / \mathrm{c}_{\mathrm{v}}$ ), which enables greater compressed gas temperatures to be achieved for fixed compression ratio configurations [12,30]. Argon has also been demonstrated to have superior heat retention characteristics relative to lighter monatomic diluent gases including helium and this minimizes heat loss to the reaction chamber walls during the ignition delay period [53]. These characteristics are due to argon's lower heat diffusivity, $\kappa$. However, this behavior is unfavorable during the wet compression process because it impedes the transport of heat to and mass from the fuel droplets in the aerosol resulting in longer evaporation times and greater thermal and/or compositional stratification within the gas phase. Neon, due in part to its lower molar mass and thus higher $\kappa$ values (nearly three 
times greater than Ar) and higher fuel mass fractions, may have some benefit for aerosol RCM experiments, especially for the most involatile liquids. This feature will be discussed in section 3.2.

For the simulations conducted in this study the droplet and gas-phase are assumed to be initially in thermal and mechanical equilibrium (i.e., uniform temperature and pressure throughout), with the liquidphase containing only fuel or water and the gas phase only oxygen and diluent. Other initialization schemes, such as assuming fuel saturation throughout the gas phase (e.g., at the saturation pressure for the initial temperature), which may be a more realistic representation of an operating aerosol RCM where some fuel evaporation could be achieved during delivery of the aerosol to the RCM, are discussed in ref. [18]. A piston trajectory for a typical RCM [29] is used to specify the time-varying volume of the gasphase computational mesh. A maximum compression ratio of $C R_{\max }=13.4$ is used with an overall compression time of $\Delta t_{\text {comp }}=15.3 \mathrm{~ms}$. It should be noted that for all of the cases studied here the evaporation process is completed before the maximum compression ratio, or overall compression time is reached. Specifying these operational parameters is important however, especially the compression time, because they affect the trajectory of the compression process, and thus the volume history which regulates droplet vaporization. The volume trace employed for this study is illustrated in Fig. 2 along with representative curves for the temperature and pressure as well as the instantaneous compression ratio. For this example an initial temperature and pressure of $T_{0}=350 \mathrm{~K}$ and $P_{0}=0.44 \mathrm{bar}$, respectively are used with a prevaporized mixture of $n$-dodecane and 'air' (i.e., $21 \% \mathrm{O}_{2}, 79 \% \mathrm{~N}_{2}$ ) at an overall equivalence ratio of $\varphi$ $=1.0$. Under conditions of no chemical reaction a compressed temperature and pressure near $T_{\text {comp }}=$ $790 \mathrm{~K}$ and $P_{\text {comp }}=13.3 \mathrm{bar}$, respectively, results for this case. For a chemically reacting case, ignition is predicted here using the detailed kinetic mechanism of Westbrook et al. [54] (2115 species, 8157 reactions). Here it can be seen that significant low temperature heat release (LTHR) occurs just before maximum compression is reached, with the second-stage heat release occurring shortly after this. While there is some uncertainty concerning the first and second stage ignition timings predicted by the Westbrook et al. mechanism, it is apparent from Fig. 2 that during aerosol RCM tests using similarly reactive fuels where the gas-phase mixture is prepared in-situ, vaporization and diffusive mixing must be 
completed well in advance of conditions where chemical reactivity becomes sufficiently rapid. This issue is discussed shortly. Evident in Fig. 2 is that the pressure rise during compression is most significant during the later stage of the compression process; the rise in temperature follows a similar non-linear trend, however this curvature is not as steep. These features significantly affect the evaporation process for some fuels and operating conditions where substantial volumetric compression can be required to achieve complete fuel evaporation (e.g., $\varphi=2.0$ ) [18]. For such conditions there is a large corresponding reduction in the reaction chamber volume which can lead to high ratios of surface area to volume and thus high levels of heat loss from the test gases. In addition, flows of the aerosol into the piston's machined crevice regions or ringpack can become significant at high $C R$ [29] and this can alter the thermodynamic state of the compressed charge, as well as contaminate the surfaces of, or gap into the machined crevice volume. The non-linearity of the temperature rise also affects gas-phase stratification during aerosol operation, as the time available for heat and mass transport to and from the droplet, respectively, becomes shorter for successive increments in compression heating. This is particularly important for fuels with very high boiling points such as n-eicosane, or very large $L_{f}$, such as water.

\subsection{Fuels and water}

\section{$\underline{3.1 .1 \text { Identical initial conditions }}$}

The fuels and water considered in this study were chosen to have significantly different boiling characteristics (see Table 1). Because of this, the $n$-heptane droplets, which have the highest vapor pressure of this group, are able to evaporate and diffuse across the gas phase much more rapidly than the other liquids included here, when initiated from identical conditions. This feature can be seen in Figure 3 where non-dimensional surface areas, $\left(d / d_{0}\right)^{2}$, for $d_{0}=3$ and $8 \mu \mathrm{m}$ droplets of the six liquids in 'air' are plotted as a function of time. An initial temperature and pressure of $T_{0}=350 \mathrm{~K}$ and $P_{0}=1 \mathrm{bar}$, respectively, are used here with an overall equivalence ratio of $\varphi=1.0$; an overall mass ratio of $\left(m_{w} / m_{a}\right)=$ 0.066 is specified for the water-'air' case. A temperature of $T_{0}=350 \mathrm{~K}$ is selected because it is within the 
heating limit of typical RCMs (e.g., $T_{\text {limit }} \sim 375 \mathrm{~K}$ due to seal degradation issues), while the initial pressure of $P_{0}=1.0$ bar results in compressed pressures near $P_{\text {comp }}=28$ bar which are relevant to diesel engine operation. The initial droplet sizes are based on previous investigations with the wet compression model where it was found that complete vaporization can be achieved for $n$-dodecane droplets from these initial conditions before a low temperature chemistry limit, chosen as $T \sim 500 \mathrm{~K}$, is reached by the gas phase $[17,18]$. This temperature is considered to be a threshold above which fuel reactivity can become significant on the time scale of the compression and evaporation processes.

In Fig. 3 it is seen that typical $d^{2}$-law behavior is not observed for any of the droplets. This is due to various features which include evaporative cooling of the droplet and gas-phase fuel saturation at the 'farfield', as well as the fact that the vaporization process is long enough so that it is affected by the gasphase compression heating process, as discussed in detail in Refs. [17,18]. It should be stressed that these evaporation simulations are different from typical droplet studies where cool droplets are introduced into elevated temperature environments, such as in an operating engine or continuous flow vaporization rig [55], where the droplets undergo initial heating and sometimes volumetric expansion to their respective boiling points. Among the five hydrocarbons investigated here, $n$-eicosane, due to its extremely low saturation pressure and corresponding high boiling temperature, takes the longest time and thus the most volumetric compression to completely evaporate. The $n$-eicosane droplets, unlike the other liquids used, initially experience an increase in surface area during wet compression because of its low volatility where the liquid-phase density decreases without a significant corresponding increase in saturation pressure, i.e., evaporation rate. After sufficient compression heating is achieved the evaporation rates counteract the liquid-phase density effects and the volumes of the droplets decrease. The water droplets, which have a high saturation pressure but also a very high vaporization enthalpy require significant extents of compression heating to overcome their $L$.

It should be noted that the initial Knudsen numbers $\left(K n_{0}=\eta / r_{s, 0}\right)$ for all of the hydrocarbons at the two droplet sizes, $d_{0}=3$ and $8 \mu \mathrm{m}$, are on the order of 0.02 and 0.007 , respectively. Knudsen numbers for the water droplets are slightly higher, near 0.08 and 0.03 , respectively. (The mean free path is computed 
using $\eta^{2}=\left(4 D_{g, f}\right)^{2} / \pi \mathrm{v}^{2}$ rms where $\mathrm{v}_{\mathrm{rms}}$ is the root mean squared velocity of the vaporized fuel at the droplet surface; this is taken to be proportional to $\sqrt{ } T_{l, r s}$.) These values are close to the Knudsen transition regime where gas kinetic effects become important $(0.1<K n<10)$, however the prescription of thermodynamic equilibria at the droplet surface has been shown to yield only modest differences $(+5-15 \%)$ in predicted evaporation times relative to more refined modeling approaches which take these effects into account [18].

Issues of fuel reactivity that result from compression heating, which are on the time scale of piston compression, are illustrated in Fig. 4 where data from an RCEM (rapid compression expansion machine) $[56,57]$ using a mixture of prevaporized n-dodecane and 'air' is presented. (Note that in an RCEM the piston does not lock at maximum compression but is free to expand the mixture.) In this figure the mean gas temperature of the mixture is plotted as function of normalized inverse volume, or instantaneous effective compression ratio $\left(C R_{\text {eff }}\right)$, which is defined as

$C R_{e f f}(t)=\frac{V_{0}}{V(t)} \frac{m(t)}{m_{0}}$

This definition accounts for mass transfer into the piston's crevice / rinpack volume. The fuel loading in 'air' for this experiment is $\varphi \sim 0.16$, which is fairly low due to challenges associated with fuel saturation / condensation at the initial temperature and pressure of $T_{0}=353 \mathrm{~K}$ and $P_{0}=1 \mathrm{bar}$, respectively. In the experimental record the LTHR and second stage heat release are identified where it can be seen that these occur during the compression stroke of the piston, well before maximum compression is achieved. LTHR begins near $T \sim 740 \mathrm{~K}$ with the second stage near $T \sim 975 \mathrm{~K}$. In this experiment the second stage heat release is not very rapid due to the low fuel loading, and a slower 'third-stage' of reaction is also observed. Similar 'third-stages' of ignition have been reported for other fuels under analogous RCM conditions [58] and during HCCI engine operation [59-60]. For reactive mixtures such as this $n$-dodecane + 'air' charge, where low temperature chemistry and heat release are significant, which is typical of high cetane number $(\mathrm{CN})$ diesel components and blends, it may not be possible to acquire conventional RCM data sets (i.e., ignition delay time as a function of compressed charge temperature at $C R_{\max }$ ) at high 
pressure and high oxygen concentrations due to 'pre-ignition' issues (see also ref. [12] for a discussion of this). However, an integrated analysis of time-resolved pressure and volume data, as depicted here, could be utilized as a validation target for chemical kinetic mechanism development (e.g., see refs. [56,57]) where discrepancies associated with LTHR timing could be highlighted and mechanism improvements made. The supplementary material provided for this article reviews the methodology employed for this figure. Evaporative cooling effects due to wet compression could easily be taken into account with this approach through the use of a physics-based sub-model for the vaporization process and its associated enthalpy transfer. Alternatively, an effective volume expansion could be used to account for evaporative cooling, while a one-dimensional analysis, similar to that developed in ref. [28] could be used to assess issues of compositional stratification due to condensation near the reaction chamber walls. In any case, during aerosol fueling and in-situ gas-phase mixture formation it will be critical that the evaporation and diffusive mixing processes are completed well before low temperature chemical reaction becomes significant. In Fig. 4 simulation results (using the wet compression model) for the $d_{0}=8 \mu \mathrm{m} n$ hexadecane droplet (cf. Fig. 3) are also presented where the initial temperature and pressure are $T_{0}=$ $350 \mathrm{~K}$ and $P_{0}=1$ bar, respectively, with $\varphi=1.0$. Computed surface and 'far-field' temperatures are shown in this figure. Here it can be seen that for the conditions utilized here (e.g., $\Delta \mathrm{t}_{\text {comp }} \sim 15 \mathrm{~ms}$ ) this very involatile hydrocarbon is predicted to evaporate by $T_{g} \sim 550 \mathrm{~K}$, with approximately $50 \mathrm{~K}$ of evaporative cooling realized.

This comparison is encouraging towards utilizing high molecular weight diesel-representative components in an aerosol RCM; however, localized compositional and thermal stratification can develop across the gas-phase. This feature can be seen in Fig. 5 where the local fuel equivalence ratios (and normalized water mass fractions), and the temperatures at the end of the evaporation process are plotted as a function of radial position for the five fuels and water at the $d_{0}=8 \mu \mathrm{m}$ size using the initial conditions from Fig. 3. The water mass fractions are normalized in Fig. 5a against the overall water to 'air' mass fraction, e.g., 0.066. For the least volatile fuels and water, the locations of 'far-field' at the end of evaporation are closest to the origin (i.e., the droplet surface) because these liquids require the most 
volumetric compression to achieve complete evaporation. These liquids also experience the greatest degree of stratification, primarily compositional, due in part to the non-linearity of the temperature rise during compression where the time available for transport of fuel from, and heat to the droplets is reduced relative to the time taken for volumetric compression. Here it can be seen that, while most of the gas phase is fairly uniform, extremely fuel-rich conditions exist near the droplet surface for some of the hydrocarbons. For example, $\varphi_{\mathrm{s}} \sim 7$ for $n$-hexadecane + 'air' and $\varphi_{\mathrm{s}} \sim 11$ for $n$-eicosane + 'air'. This condition could be problematic during RCM operation where fuel pyrolysis at the elevated temperatures and pressures may lead to locally non-homogeneous or early ignition behavior. Water exhibits substantially different behavior relative to the hydrocarbons where there is a low degree of compositional stratification across the gas phase but significant thermal stratification exists, as can be seen in Fig. 5 b. This is due to the difference in Lewis numbers between the hydrocarbons and water, as well as the more significant $L$ required for the water evaporation. These issues, and effects of other fuel and diluent properties on the development of stratification during wet compression, are discussed in more detail in the next section. Though not indicated in the previous figures, it should be noted that the stratification shown in Fig. 5 is predicted to require approximately $0.1-0.5 \mathrm{~ms}$ after the end of evaporation to completely dissipate. The diffusion, primarily of fuel for the hydrocarbons, is relatively rapid compared to the overall evaporation time and should yield a locally homogeneous mixture by the time that the test conditions are reached; however, it would be beneficial if such large gradients can be avoided during the wet compression process.

\subsubsection{Identical initial vaporization rates}

It is evident from the previous section that the boiling curve of a particular fuel is extremely important towards enabling its successful utilization in an aerosol RCM experiment. It is also critical however, to understand the influence of gas-phase diffusion and the effects of fuel (and water) transport properties, on the gas-phase stratification that can result during wet compression. These features are isolated in this section. To conduct this investigation, the initial temperatures of the droplets are adjusted so that the 
initial equilibrium concentrations of fuel and water at the droplet surface at the start of the experiment, and the corresponding initial evaporation rates are similar. Under these conditions mass transport to the 'far-field' is dependent primarily on the density-weighted mass diffusivity, $\rho_{g} D_{g}$. A value of $Y_{f, s} \sim$ $0.042 \mathrm{~g}_{f} / \mathrm{g}_{\text {mix }}$ is selected for these simulations because this value is the saturated fuel mass fraction for $n$ dodecane in 'air' at $T_{0}=350 \mathrm{~K}$ and $P_{0}=1.0 \mathrm{bar}$. (In the simulations the specified fuel concentrations at the droplet surface are achieved shortly after the calculations are initialized from the zero gas-phase fuel concentration condition.)

Figures 6-9 present select results for five of the liquids evaporating into 'air' at the two droplet sizes from the initial conditions listed in Table $1 ; n$-eicosane is not used for these runs due to its poor performance illustrated in Section 3.1.1, though its properties at the conditions of this test are included in Table 1 for reference. Figure 6 illustrates the behavior of the non-dimensional surface areas, $\left(d / d_{0}\right)^{2}$, as a function of time, while Fig. 7 shows the non-dimensional evaporation rates, defined as $\bar{m}=\dot{m} / 4 \pi \rho_{g} D_{g} r_{s}$, as a function of non-dimensional time, which is defined as $\bar{t}=\int \hat{\kappa}_{l} d t / r_{s}{ }^{2}$. Changes in $\rho_{g}, D_{g}$, and $\hat{\kappa}_{l}$ with time (i.e., $T, P$ ) are included in the calculation of $\bar{m}$ where these effects are most noticeable at long times and for the largest droplets, though the trends are identical if constant values for these properties are used. In Fig. 6 it is evident that under these conditions, at both droplet sizes, the fastest evaporation is achieved by the $n$-hexadecane droplets followed by HMN, $n$-dodecane, $n$-heptane, and then water. This shifted hierarchy is primarily due to the differences in vaporization enthalpies and the resulting evaporative cooling that occurs, where greater compression heating is required to overcome this. Water, which has $L$ many times greater than the four hydrocarbons, requires significantly greater volumetric compression in order to achieve complete vaporization of the liquid droplets; this feature was also seen in Fig. 3 . The $d_{0}$ $=8 \mu \mathrm{m}$ water droplet in Fig. 6 in fact requires nearly the entire compression stroke, to $C R \sim 13.3$, to evaporate. The difference in evaporation times between $n$-hexadecane and HMN, where $n$-hexadecane has $L_{f}$ larger than HMN, is due to the slightly higher value of $\rho_{g} D_{g}$, and slightly lower $\rho_{l 0}$ as indicated in 
Table 1, where these enable the smaller amount of $n$-hexadecane to be evaporated and transported away from the droplet more effectively.

Issues of evaporative cooling and 'far-field' saturation are illustrated in Fig. 7. Due to the initial prescription of similar values for $Y_{f, s}$ for all of the liquids, the initial non-dimensional evaporation rates are observed to be very similar. In addition, it can be seen that as the vaporization process progresses, all of the flow rates are seen to decrease; this is due to the evaporative cooling that occurs at the droplet surface, as discussed in Refs. $[17,18]$. None of these droplets experience volumetric expansion, as observed with the $n$-eicosane droplets in Fig. 3. In Fig. 7 it is evident that the greatest drop in evaporation rate (before $\bar{t} \sim 7$ ) is experienced by the water droplets followed by $n$-heptane, $n$-dodecane, $n$ hexadecane and finally HMN. This trend is due to the difference in $L$ among the droplets. In general, the relative decrease in initial evaporation rates seen among the five liquids results in the difference in overall evaporation times discussed in Fig. 6. Another feature that is evident in Fig. 7 is that the $d_{0}=3 \mu \mathrm{m}$ droplets experience a greater decrease in $\bar{m}$, relative to the $d_{0}=8 \mu \mathrm{m}$ droplets; this is due to 'far-field' saturation effects. Smaller droplets at the same overall $\varphi$ have shorter diffusion distances across which the fuels and water must travel to reach the 'far-field'; the 'far-field' therefore saturates more quickly for smaller droplets and correspondingly slows their vaporization rates [18]. This characteristic is responsible for the differences in the evaporation trajectories seen between the two droplet sizes in Fig. 5 for all of the liquids.

Figures 8 and 9 illustrate the gas-phase fuel and water mass fractions, and the temperatures, respectively, at the droplet surface and at the 'far-field' as a function of non-dimensional surface area. Again, there is no liquid-phase compositional or thermal stratification due to the small sizes of the droplets utilized. In Fig. 8 the approximate corresponding equivalence ratios for the fuels are labeled on the right vertical axis. In Fig. $8 \mathrm{a}$ the $d_{0}=3 \mu \mathrm{m}$ cases are shown while Fig. $8 \mathrm{~b}$ presents the $d_{0}=8 \mu \mathrm{m}$ cases. In both images it can be seen that during the initial period of evaporation the mass fractions at the droplet surface decrease; this is due to evaporative cooling. The water droplets experience the most significant decrease in $Y_{f, s}$ because this liquid has the largest $L$. Also noticeable in these two figures is that the 
smaller droplets have greater degrees of gas-phase saturation, meaning that the 'far-field' fuel and water concentrations are much closer to the saturated conditions at droplet surfaces. For the $d_{0}=3 \mu \mathrm{m}$ water droplet in fact, it can be seen that the gas-phase becomes completely saturated at $\left(d / d_{0}\right)^{2} \sim 0.875$; additional volumetric compression drives the mixture beyond this point. Finally, in comparing all five liquids it can be seen that water vaporization yields a much more compositionally uniform gas phase than for the hydrocarbons. The stratification for the fuels is most significant for HMN, followed by $n$ hexadecane, $n$-dodecane and $n$-heptane. These features are due to the differences in magnitude of $\rho_{g} D_{g}$, as indicated in Table 1.

Figure 9 illustrates the differences in thermal behavior of the gas phase for the four fuels and water. Here issues of evaporative cooling, compression heating and thermal stratification are visible. While not specifically identified for all of the liquids in the figure, the lower temperatures presented here are at the droplet surface while the higher temperatures are at the 'far-field'. As discussed already, the gas phase for the small droplets experiences a greater drop in temperature than for the larger droplets, where, similar to fuel transport to the 'far-field', this is due to the shorter diffusion distance that the heat must travel from the 'far-field' to reach the droplets. The smaller droplets yield a lower degree of stratification during the wet compression process than the larger ones. The water droplets and surrounding gas experience the greatest drop in temperature during vaporization due to its higher vaporization enthalpy. The water droplets also experience a much greater degree of thermal stratification than the hydrocarbons. This is due to the lower Lewis number of the mixture $\left(L e_{g}=\kappa_{g} / D_{g}<1.0\right)$ and the very large vaporization enthalpy where the evaporated water is transported away quickly from the droplet, thereby facilitating the evaporation process, however the substantial amount of required heat is not supplied as readily. This is especially significant for the $d_{0}=8 \mu \mathrm{m}$ droplet which experiences a thermal gradient of $\sim 150 \mathrm{~K}$ across the gas phase at the completion of evaporation. The hydrocarbons, conversely, all have fairly small degrees of thermal stratification while their compositional stratification is much more significant. Either form of stratification is of concern during RCM experiments, especially if this is not able to dissipate before chemical reactivity becomes significant, as discussed in the previous section. Overall, for all of the 
liquids investigated, the smaller droplets result in gas-phase conditions that are much more uniform, both compositionally and thermally; for aerosol RCM experiments this feature is extremely desirable.

Figure 10 illustrates the compositional and thermal stratification that exists across the gas phase at the end of evaporation for the $d_{0}=8 \mu \mathrm{m}$ droplets from Figs. $8 \mathrm{~b}$ and $9 \mathrm{~b}$. Here the equivalence ratios for the four fuels are presented as a function of radial position while water mass fractions, similar to Fig. 5, are normalized to $\left(m_{w} / m_{a}\right)=0.066$; i.e., the overall mass ratio. As in Fig. 5 it can be seen that most of the gas-phase is fairly uniform with the largest fuel concentrations located close to the droplets. For the water case there is significant thermal stratification across the gas phase.

Figure 11 summarizes the results of these fuel simulations. Here 'operating maps' are constructed for $n$-dodecane [18] and $n$-hexadecane; these 'maps' cover a wider range of initial droplet diameters and overall fuel loadings than shown in the previous figures. Contours are drawn which indicate the compression ratio, peak gas-phase temperature and maximum local equivalence ratio at the end of the evaporation process. Nitrogen is used as the diluent. The operating regimes which have a peak temperature below $500 \mathrm{~K}$ are shaded, where this indicates the capability to completely vaporize the fuel droplets before the low temperature chemistry threshold is reached. Also shown on the $n$-hexadecane 'map' is the regime where the maximum piston compression is reached in the RCM, i.e., maximum CR, before vaporization is completed. It should be highlighted that very large equivalence ratios can be experienced for the large droplet diameters, where this can lead to premature fuel reactivity near the droplet surface, as already discussed.

\subsection{Diluent gases}

It is important to understand the influence of diluent gas properties on the wet compression process, as there may be opportunities to tailor the gas-phase mixture composition for particular fuels, e.g., to enhance evaporation of extremely involatile liquids beyond $\sim$ n-hexadecane, such as biodiesel. The diluent gases considered here have different heat capacities and transport characteristics where representative properties are listed in Table 2. Argon is often used as a monatomic diluent in ST and 
RCM experiments, however neon may provide some benefit within wet compression experiments due to its more favorable transport characteristics which can enhance the wet compression process. Helium is not considered in this study due to problems that have been highlighted concerning significant devicescale heat loss and thermal stratification that develops across the reaction chamber during the test period, as discussed in ref. [53]. The initial temperature and pressure conditions utilized for these simulations, as in section 3.1.1, are consistent for each oxygen + diluent gas mixture where these are set to $T_{0}=350 \mathrm{~K}$ and $P_{0}=1.0$ bar, with an overall fuel loading of $\varphi=1.0 . n$-Dodecane is used as the fuel while similar trends are seen with the other hydrocarbons. Oxygen mole fractions are maintained at $21 \%$ of the initial gasphase composition and droplet sizes of $d_{0}=3$ and $8 \mu \mathrm{m}$ are considered.

Figure 12 presents the non-dimensional surface areas for these cases plotted as a function of time. Under these conditions, it can be seen that both monoatomic diluent gases yield more rapid rates of evaporation, and thus shorter overall vaporization times. This behavior is due primarily to their lower heat capacities (see Table 2) and therefore greater increases in temperature that occur upon volumetric compression. This feature is discussed more with regard to Fig. 15. Also evident in Fig. 12 is that the neon mixtures result in more rapid vaporization than the argon mixtures; evaporation times are $\sim 12 \%$ faster. This characteristic is due to differences in the gas-phase transport of mass from and heat to the droplet. Representative density-weighted mass diffusivities are listed in Table 2, and although it can be seen that the argon mixture has a greater value of $\rho_{g} D_{g}$ than the neon mixture, the mass fraction of fuel vapor at the droplet surface in the neon case is much larger due to the lower molar mass of that diluent gas. This is evident in Figure 13 where the fuel mass fractions at the droplet surface and at the 'far-field' are plotted as a function of non-dimensional surface area for the $d_{0}=3 \mu \mathrm{m}$ droplet. The larger gradient of fuel mass fraction across the gas phase for the neon mixture, due to neon's lower molar mass, enables more rapid diffusion of fuel away from the droplet and to the 'far-field'. Similarly, heat is able to diffuse to the droplet more effectively in the neon case, as can be seen in Figs. 14 and 15. 
In Figs. 14 and 15 the effects of different heat transport and storage characteristics on the wet compression process for the three diluent gases are illustrated. Figure 14 presents the temperatures at the droplet surface and at the 'far-field' as a function of non-dimensional surface area for the two droplet sizes. Here it can be seen that the temperature of the neon mixture is the lowest at the end of the evaporation process, with this followed by the nitrogen and then the argon mixtures. This feature is due in part to the greater thermal diffusivity of the neon mixture (which is twice as high as the other two), and the more rapid rise in temperature of the neon and argon mixtures relative to nitrogen. The higher thermal diffusivity of the neon mixture enables more effective transport of the heat required for vaporization to be transported to the droplet; this also yields a lower level of thermal stratification across the gas phase than is observed in the argon mixture.

Figure 15 illustrates the temperature history of the gas phase at the droplet surface and at the 'farfield' as a function of instantaneous compression ratio. In this figure the trajectories are shifted for the neon $(T+40 \mathrm{~K})$ and argon $(T+20 \mathrm{~K})$ mixtures for effective visualization. Here it is readily evident that the temperature rise as a function of compressed volume is greater for the monoatomic gases due to their lower heat capacities. This, in general results in less required volumetric compression of the aerosol in order to achieve droplet vaporization. This characteristic could be important for large $L_{f}$ fuels or conditions of high fuel loading (e.g., see ref. [18] and Fig. 12). In addition, during RCM operation this feature is beneficial because, as discussed in section 3.1.1, heat loss is smaller if lower peak compression ratios can be used due to lower surface area to volume ratios. There is also a lower potential for unvaporized droplets of the aerosol to enter, and/or contaminate the piston's machined crevice regions or ringpack at smaller $C R$.

Overall it can be seen that neon as a diluent gas species may offer benefits relative to argon during aerosol RCM operation due to its lower molar mass and thus higher heat and mass transport characteristics. This is contrary to conventional RCM operation. These properties enhance the wet compression process, and may be especially favorable for extremely involatile, diesel-representative hydrocarbons and oxygenated hydrocarbons. Though not presented here, an analogous simulation using a 
$d_{0}=8 \mu \mathrm{m}, n$-hexadecane droplet with $T_{0}=350 \mathrm{~K}$ and $P_{0}=1.0$ bar indicates similar trends for the higher boiling point hydrocarbons. The compression ratio required for complete evaporation can be reduced from 4.3 to 2.4 if neon is utilized instead of nitrogen, and the resulting localized fuel stratification is lower relative to the case where argon is used as the diluent, e.g., $\varphi_{s, \mathrm{Ne}}=6.7$ compared to $\varphi_{s, \mathrm{Ar}}=8.3$ near the end of evaporation. However, bulk scale transport of heat to the cold reaction chamber walls could be enhanced with neon [53] and the potential detrimental effects of this diluent characteristic need to be investigated. This is particularly of interest because it is expected that the aerosol delivery process (to the RCM's reaction chamber from an externally generated aerosol) will result in substantial residual fluid dynamics in the reaction chamber relative to a conventionally prepared RCM experiment, and this could increase heat loss from the test gases.

\section{Summary}

An unsteady spherically-symmetric, single-droplet wet compression model is used to investigate fuel and diluent property effects during wet compression of a fuel aerosol under RCM conditions. $n$-Heptane, $n$-dodecane, 2,2,4,4,6,8,8-heptamethylnonane (isocetane), $n$-hexadecane (cetane) and $n$-eicosane are employed as diesel-representative fuels, while comparisons are made to water droplets. Nitrogen, neon and argon are selected as the gas-phase diluents while the oxidizer is considered to be oxygen at atmospheric concentrations. The compression time is set to $\Delta t_{\text {comp }} \sim 15 \mathrm{~ms}$ while the geometric compression ratio is fixed at $C R=13.4$. It is demonstrated in this study that under these conditions involatile fuels up to $\sim n$-hexadecane appear to have potential as candidates for aerosol RCM experiments. To achieve complete vaporization and adequate dissipation of localized compositional and thermal stratification in advance of significant low temperature chemical reactivity however, very small droplets $\left(d_{0}<5 \mu \mathrm{m}\right)$ and some pre-heating are expected to be required. Fuels with much lower saturation pressures (e.g., $n$-eicosane) might not be usable unless extremely small droplets $\left(d_{0}<1 \mu \mathrm{m}\right)$, longer compression times (e.g., $\Delta t_{\text {comp }} \sim 30 \mathrm{~ms}$ ) and/or lower overall pressures (e.g., $P_{0} \sim 0.5$ bar), see ref. [17], 
are employed to achieve adequate vaporization and gas-phase uniformity. Such conditions should be explored, especially towards utilizing soy or rapeseed derived biodiesel components (e.g., methyl oleate) in RCMs. Also determined through this work is that the boiling curve and $L_{f}$ are the dominant fuel and water properties while the density-weighted mass diffusivity, $\rho_{g} D_{g}$, generally plays a secondary role. The heat capacity and molar mass are the dominant diluent properties that affect the near-droplet and 'farfield' conditions. The magnitude of the gas-phase mixture Lewis number contributes to either greater compositional $\left(L e_{g}>1\right)$ or thermal $\left(L e_{g}<1\right)$ stratification. For large hydrocarbons and oxygenated hydrocarbons that are representative of diesel fuels, compositional stratification can be large and will be considerably greater than thermal stratification. This characteristic has the potential to undesirably alter the ignition/oxidation data from aerosol RCMs because fuel pyrolization could occur at extremely fuelrich conditions $\left(\varphi_{\mathrm{s}}>5\right)$ near the droplet surface before vaporization is complete. Neon, due in part to its lower molar mass and thus greater thermal and mass transport capabilities, may have potential benefits over argon as a diluent gas for aerosol RCM experiments, especially for larger $T_{\text {boil }}$ liquids. Faster evaporation could be achieved while localized compositional and thermal stratification are reduced. Issues of increased device-scale heat loss however, need to be explored.

\section{Acknowledgements}

Professor Rolf Reitz at the University of Wisconsin Engine Research Center is thanked for his assistance in locating a copy of the original source code for the transient droplet evaporation model. Funding for this work has been provided in part through NSF OCI-0923037 and CBET-0968080, where the simulations were conducted using Marquette University’s Pere Computing Cluster.

\section{References}

[1] S. Kimura, O. Aoki, Y. Kitahara, and E. Aiyoshizawa, SAE Paper 2001-01-0200, 2001.

[2] H. Yanagihara, SAE Paper 2002-30-0009, 2002.

[3] H. Ogawa, N. Miyamoto, K. Akao, SAE 2002-08-0423, 2002. 
[4] S. Simescu, S.B. Fiveland, and L.G. Dodge, SAE 2003-01-0345, 2003.

[5] M. Canova, S. Midlam-Mohler, Y. Guezennec, G. Rizzoni, Int. J. Vehicle Design 44 (2007) 62-83.

[6] A.S. Cheng, B.T. Fisher, G.C. Martin and C.J. Mueller, Energy Fuels 24 (2010) 1538-1551.

[7] J.H. Mack, S.M. Aceves, and R.W. Dibble, Energy 34 (2009) 782-787.

[8] M. Chaker, C.B. Meher-Homji and T. Mee III, J. Eng. Gas Turbines Power 126 (2004) 545-580.

[9] R. Bhargava, C.B. Meher-Homji, J. Eng. Gas Turbines Power 127 (2005) 145-158.

[10] R.K. Bhargava, C.B. Meher-Homji, M.A. Chaker, M. Bianchi, F. Melinao, and A. Peretto, J. Eng. Gas Turbines Power 129 (2007) 454-472.

[11] J. Vanderover and M.A. Oehlschlaeger, Int. J. Chem. Kinet. 41 (2009) 82-91.

[12] K. Kumar and C.-J. Sung, Combust. Flame 157 (2010) 676-685.

[13] D.F. Davidson, D.R. Haylett, R.K. Hanson, Combust. Flame 155 (2008) 108-117.

[14] C. Allen, G. Mittal, C.J. Sung, E. Toulson, T. Lee, Proc. Comb. Inst. 33 (2010) 3367-3374.

[15] B. Rotavera, N. Polley, E.L. Petersen, K. Scheu, M. Crofton, G. Bourque, Proc. ASME Turbo Expo 2010, GT2010-22844, 2010.

[16] G. Mittal and C.-J. Sung, Combust. Flame 145 (2006) 160-180.

[17] M.V. Johnson, G.S. Zhu, S.K. Aggarwal, and S.S. Goldsborough, S.S., Int. J. Heat Mass Transfer 53 (2010) $1100-1111$.

[18] S.S. Goldsborough, M.V. Johnson, G.S. Zhu, and S.K. Aggarwal, Combust. Flame 158 (2011) 57-68..

[19] G.S. Zhu and S.K. Aggarwal, Int. J. Heat Mass Transfer 43 (2000) 1157-1171.

[20] G.S. Zhu, R.D. Reitz, S.K. Aggarwal, Int. J. Heat Mass Transfer 44 (2001) 3081-3093.

[21] G.S. Zhu and S.K. Aggarwal, J. Eng. Gas Turbines Power 124 (2002) 762-770.

[22] W. Li and E.J. Davis, Aerosol Sci. Tech. 25 (1996) 11-21.

[23] S.S. Sazhin, I.N. Shishkova, A.P. Kryukov, V.Yu. Levashov, M.R. Heikal, Int. J. Heat Mass Transfer 50 (2007) 2675-2691.

[24] V.R. Dushin, A.V. Kulchitskiy, V.S. Nerchenko, V.F. Nikitin, E.S. Osadchaya, Yu.G. Phylippov, N.N. Smirnov, Acta Astronautica 63 (2008) 1360-1371.

[25] S.S. Sazhin and I.N. Shishkova, Atom. Spray 19 (2009) 473-489.

[26] M.V. Johnson, MS Thesis, Marquette University, 2009. 
[27] D.Y. Peng and D.B. Robinson, Ind. Eng. Chem.: Fund. 15 (1976) 59-64.

[28] S.S. Goldsborough, C. Banyon, G. Mittal, Combust. Flame (submitted).

[29] S.S. Goldsborough and C.J. Potokar, SAE Paper 2007-01-0169, 2007.

[30] S.M. Gallagher, H.J. Curran, W.K. Metcalfe, D. Healy, J.M. Simmie, G. Bourque, Combust. Flame 153 (2008) 316-333.

[31] T.H. Chung, M. Ajlan, L.L. Lee and K.E. Starling, Ind. Eng. Chem. 27 (1988) 671-679.

[32] D. Roy and G. Thodos, Ind. Eng. Chem. Fundam. 7 (1968) 529-534.

[33] D. Roy and G. Thodos, Ind. Eng. Chem. Fundam. 9 (1970) 71-79.

[34] B.E. Poling, J.M. Prausnitz and J.P. O'Connell, The Properties of Gases and Liquids, $5^{\text {th }}$ ed., 2001.

[35] S. Takahashi, J. Chem. Eng. 6 (1974) 417-420.

[36] R.B. Bird, W.E. Stewart and E.N. Lightfoot, Transport Phenomena, $2^{\text {nd }}$ ed. John Wiley and Sons, New York, 2002.

[37] http://garfield.chem.elte.hu/burcat/burcat.html

[38] E.R. Ritter and J.W. Bozzelli, Int. J. Chem. Kinet. 23 (1991) 767-778.

[39] I.L. Byung and G.K. Michael, AIChE J. 21 (1975) 510-527.

[40] V. Rùzicka and E.S. Domalski, J. Phys. Chem. Ref. Data 22 (1993) 597-619.

[41] L.P. Filippov, Chem. Abstr. 50 (1956) 8276.

[42] K. Nakanishi, Ind. Eng. Chem Fundam. 17 (1978) 253-256.

[43] G. Watson, C.K. Zéberg-Mikkelsen, A. Baylaucq and C. Boned, J. Chem. Eng. Data 51 (2006) $112-118$.

[44] D.R. Caudwell, J.P.M. Trusler, V. Vesovic, W.A. Wakeham, Int. J. Thermophys. 25 (2004) 1572-9567.

[45] M. Barrouhou, C.K. Zéberg-Mikkelsen, A. Baylaucq, C. Boned, Int. J. Thermophys. 24 (2003) $937-952$.

[46] M.A. Matthews, J.B. Rodden and A. Akgerman, J. Chem. Eng. Data 32 (1987) 319-322.

[47] S. Chynoweth, Y. Michopoulos, Molecular Phys. 81 (1994) 133-141.

[48] G.H. Thomson, K.R. Brobst and R.W. Hankinson, AIChE J. 28 (1982) 671-676.

[49] S. Will and A. Leipertz, Int. J. Thermophys. 18 (1997) 1339-1354.

[50] J.T. Farrell, N.P. Cernansky, F.L. Dryer, D.G. Friend, C.A. Hergart, C.K. Law, R.M. McDavid, C.J. Mueller, A.K. Patel and H. Pitsch, SAE Paper 2007-01-0201, 2007.

[51] L.S. Ott and T.J. Bruno, Energy Fuels 22 (2008) 2861-2868. 
[52] C. Laurent, G. Lavergne, P. Villedieu, Int. J. Multiphase Flow 36 (2010) 51-59.

[53] J. Würmel, E.J. Silke, H.J. Curran, M.S. Ó Conaire, J.M. Simmie, Combust. Flame 151 (2007) 289-302.

[54] C.K. Westbrook, W.J. Pitz, O. Herbinet, H.J. Curran, E.J. Silke, Combust. Flame 156 (2009) 181-191.

[55] V. Duprédurand, G. Castanet and F. Lemoine, Int. J. Heat Mass Trans. 53 (2010) 3495-3502.

[56] S. Goldsborough, $5^{\text {th }}$ US Combustion Meeting, 2007.

[57] S. Goldsborough, WSSCI Fall Technical Meeting 2007.

[58] Y. Ohta and M. Furutani, Archivum Combustionis 11 (1991) 43-52.

[59] X. Lu, L. Ji, J. Ma, Z. Huang, Energy \& Fuels 21 (2007) 1468-1473.

[60] H. Machrafi and S. Cavadias, Combust Flame 155 (2008) 557-570.

[61] S. Goldsborough, Comb. Sci. Tech. 181 (2009) 729-755.

[62] G. Mittal, M.P. Raju and C.-J. Sung, Combust. Flame 155 (2008) 414-428.

[63] S. Goldsborough, SAE Transactions 116:3 (2007) 632-651.

[64] S. Goldsborough, SAE Paper 2006-01-0870, 2005.

[65] G. Woschni, SAE Paper 670931, 1967.

[66] C.M. Lund, and L. Chase, Rep. UCRL-52504, Lawrence Livermore National Laboratory, 1995.

[67] M. Ò Connaire, H.J. Curran, J.M. Simmie, W.J. Pitz, and C.K. Westbrook, Int. J. Chem. Kinet. 36 (2004) 603622.

[68] J. Li, Z. Zhao, A. Kazakov, F.L. Dryer, F.L., Int. J. Chem. Kinet. 36 (2004) 566-575.

[69] S.G. Davis, A.V. Joshi, W. Wang, F. Egolfopooulos, Proc. Comb. Inst. 30 (2005) 1283-1292.

[70] P. Saxena and F.A. Williams, Combust. Flame 145 (2006) 316-323.

[71] E. Ranzi, A. Frassoldati, S. Granata, T. Faravelli, Ind. Eng. Chem. Res. 44 (2005) 5170-5183.

[72] O. Herbinet, P.-M. Marquaire, F. Battin-Leclerc, R. Fournet, J. Anal. App. Pyrolysis 78 (2007) 419-429.

[73] H. Oshibe, H. Nakamura, T. Tezuka, S. Hasegawa, K. Maruta, Combust. Flame (2010) 1572-1580.

[74] A. Yamamoto, H. Oshibe, H. Nakamura, T. Tezuka, S. Hasegawa, K. Maruta, Proc. Combust. Inst (2011) 3259-3266.

[75] S.S. Vasu, PhD Dissertation, Stanford University, 2010. 


\section{Appendix}

This appendix discusses a sensitivity analysis of the wet compression model considering perturbations to various fuel and diluent gas properties. The intent is to understand the sensitivity of the simulation results to errors associated with the thermo-physical models, as well as to highlight which properties most influence droplet evaporation times during wet compression. The analysis is conducted by artificially modifying the thermo-physical properties, in a one-at-a-time fashion, and performing wet compression simulations using two initial droplet diameters. $n$-Dodecane is used as the fuel with nitrogen as the diluent and the initial temperature and pressure set to $T_{0}=350 \mathrm{~K}$ and $P_{0}=1 \mathrm{bar}$, respectively; the overall fuel loading is maintained at $\varphi=1.0$. Other simulation conditions are similar to those specified within the main text.

Figure A1 presents the results of this analysis where normalized sensitivity coefficients are provided for each of the properties investigated. The sensitivity coefficients are computed using

$$
S=\frac{\partial F(x)}{\partial x} \frac{x}{F(x)} \approx \frac{\Delta t_{\text {evap }}(1.15 x)-\Delta t_{\text {evap }}(0.85 x)}{1.15 x-0.85 x} \frac{x}{\Delta t_{\text {evap }}(x)}
$$

where $x$ is the property investigated and $F$ is a general function, in this case taken as $\Delta t_{\text {evap }}$, the time required to achieve complete fuel evaporation. In this formulation positive sensitivity values indicate that the property has a tendency to increase the evaporation time (i.e., slow the process down), while negative sensitivity values indicates a tendency to decrease the evaporation time (i.e., speed up the process).

From this figure it can be seen that the most sensitive fuel properties are $\rho_{l, f}, L_{f}, D_{g, f}, \phi_{f}^{l}$, while $c_{p g, d i l}$ and $\alpha_{g, d i l}$ are the most sensitive diluent gas properties. The fuel density and vaporization enthalpy have positive sensitivities along with the diluent gas heat capacity. This is because as the liquid phase density increases more fuel must be vaporized (since the droplet size is kept constant here); as the vaporization enthalpy increases more heat, i.e., compression work, must be supplied for vaporization; as the diluent gas heat capacity increases the temperature achieved through volumetric compression is reduced, while the thermal diffusivity of the gas phase also decreases. The fuel diffusion and fugacity coefficients have negative sensitivities along with the diluent gas thermal conductivity. This is due to the fact that as the 
diffusion coefficient increases the fuel can be transported more easily away from the droplet; as the liquid phase fugacity coefficient increases the equilibrium condition favors more fuel in the vapor phase at the droplet surface; as the diluent gas thermal conductivity increases heat can more effectively be transported to the droplet surface. It should be noted that for the small perturbations employed here $( \pm 15 \%$ of the fuel or diluent gas property), the relative change in total evaporation times are on the order of $\pm 6.5 \%( \pm 0.4 \mathrm{~ms})$ for the $d_{0}=3 \mu \mathrm{m}$ droplet, and $\pm 3.5 \%( \pm 0.3 \mathrm{~ms})$ for the $d_{0}=8 \mu \mathrm{m}$ droplet. The change in evaporation time for the $d_{0}=3 \mu \mathrm{m}$ droplet at the $\pm 15 \% c_{p g, d i l}$ conditions is a bit higher, $\pm 30 \%$.

It is interesting to note that $\alpha_{l, f}$ displays virtually no sensitivity for the $d_{0}=3$ or $8 \mu \mathrm{m}$ droplets investigated here; for larger droplets (e.g., $\left.d_{0} \sim 18 \mu \mathrm{m}\right)$ the sensitivity is slightly positive $(S=+0.003)$. This positive value is due to the increase in heat transport away from the droplet surface, i.e., to the droplet center, and thus a reduction in the surface temperature (and corresponding saturation pressure). This result seems to contradict the findings of ref. [14] where the authors of that paper concluded that increased fuel droplet thermal conductivity $(+3600 \%)$, due to the inclusion of aluminum nano-particles ( $2 \%$ by weight), leads to faster rates of fuel vaporization and thus shorter ignition delay times, relative to undoped fuels in aerosol RCM experiments. Based on our sensitivity analysis, it appears more probable that other factors, such as the increased gas-phase thermal diffusivity $(+30 \%)$ or perhaps enhanced chemical processes, are responsible for significant differences in measured ignition delay times between nano-particle laden fuels and undoped ones under wet compression conditions. 


\section{Figure Captions}

Figure 1 - Schematic of wet compression model illustrating liquid- and gas-phase domains and the location of the 'far-field' boundary.

Figure 2 - Representative volume, compression ratio, temperature and pressure histories used in this study where the volume data is typical of rapid compression machines described in the literature. The temperature and pressure trajectories are computed assuming an adiabatic compression process from $T_{0}=$ $350 \mathrm{~K}$ and $P_{0}=0.44 \mathrm{bar}$, respectively, with $\varphi=1.0$ using a fully pre-vaporized / pre-mixed charge. Nonreacting and reacting temperature profiles are shown where the reacting case is computed based on the detailed kinetic mechanism of Westbrook et al. [54].

Figure 3 - Non-dimensional droplet surface areas as a function of time for various fuels and water in oxygen + nitrogen at an overall stoichiometric ratio $(\varphi=1.0)$ for droplet sizes of $d_{0}=3$ and $8 \mu \mathrm{m}$ from an initial temperature and pressure of $T_{0}=350 \mathrm{~K}$ and $P_{0}=1$ bar, respectively.

Figure 4 - Comparison of RCEM mean gas temperature for a pre-vaporized $n$-dodecane + 'air' mixture with simulation results of the wet compression model using an unvaporized $n$-hexadecane + 'air' mixture. The initial temperatures for both cases are near $T_{0} \sim 350 \mathrm{~K}$ with initial pressures of $P_{0}=1$ bar. Also indicated here is the adiabatic-core temperature for the $n$-dodecane + 'air' mixture (no heat release) to illustrate the extent of evaporative cooling that occurs during wet compression at this fuel loading.

Figure 5a-Gas-phase fuel equivalence ratios and normalized water mass fraction at the end of evaporation as a function of radial position for various fuels and water in oxygen + nitrogen at an overall 
stoichiometric ratio $(\varphi=1.0)$ for a droplet size of $d_{0}=8 \mu \mathrm{m}$ using an initial temperature and pressure of $T_{0}$ $=350 \mathrm{~K}$ and $P_{0}=1$ bar, respectively.

Figure 5b - Gas-phase temperatures as a function of radial position at the end of evaporation for various fuels and water in oxygen + nitrogen at an overall stoichiometric ratio $(\varphi=1.0)$ for a droplet size of $d_{0}=$ $8 \mu \mathrm{m}$ using an initial temperature and pressure of $T_{0}=350 \mathrm{~K}$ and $P_{0}=1 \mathrm{bar}$, respectively.

Figure 6 - Non-dimensional droplet surface areas as a function of time for various fuels and water in oxygen + nitrogen at an overall stoichiometric ratio $(\varphi=1.0)$ for droplet sizes of $d_{0}=3$ and $8 \mu \mathrm{m}$ from the initial conditions listed in Table 1, where different initial temperatures are used.

Figure 7 - Non-dimensional evaporation rates as a function of non-dimensional time for various fuels and water in oxygen + nitrogen at an overall stoichiometric ratio $(\varphi=1.0)$ for droplet sizes of $d_{0}=3$ and $8 \mu \mathrm{m}$ from the initial conditions listed in Table 1, where different initial temperatures are used.

Figure 8 - Gas-phase mass fractions of fuel (water) at the droplet surface and at the 'far-field' as a function of non-dimensional surface area for various fuels and water in oxygen + nitrogen at an overall stoichiometric ratio $(\varphi=1.0)$ for droplet sizes of $d_{0}=3$ and $8 \mu \mathrm{m}$ from the initial conditions listed in Table 1 , where different initial temperatures are used.

Figure 9 - Gas-phase temperatures at the droplet surface and at the 'far-field' as a function of nondimensional surface area for various fuels and water in oxygen + nitrogen at an overall stoichiometric ratio $(\varphi=1.0)$ for droplet sizes of $d_{0}=3$ and $8 \mu \mathrm{m}$ from the initial conditions listed in Table 1 , where different initial temperatures are used. 
Figure 10 - Gas-phase fuel equivalence ratios and normalized water mass fractions, and temperatures at the end of evaporation as a function of radial position for various fuels and water in oxygen + nitrogen at an overall stoichiometric ratio $(\varphi=1.0)$ for a droplet size of $d_{0}=8 \mu \mathrm{m}$ from the initial conditions listed in Table 1, where different initial temperatures are used.

Figure 11 - Predicted 'operating maps' for wet compression of (a) $n$-dodecane + oxygen + nitrogen and (b) $n$-hexadecane + oxygen + nitrogen where the compression ratio, peak gas-phase temperature and peak equivalence ratio at the end of the vaporization process are indicated for a range of initial droplet diameters at various overall fuel loadings.

Figure 12 - Non-dimensional droplet surface areas as a function of time for $n$-dodecane + oxygen and various diluents at an overall stoichiometric ratio $(\varphi=1.0)$ for droplet sizes of $d_{0}=3$ and $8 \mu \mathrm{m}$ from $T_{0}=$ $350 \mathrm{~K}, P_{0}=1$ bar.

Figure 13 - Gas-phase fuel mass fractions at the droplet surface and at the 'far-field' as a function of non-dimensional surface area for $n$-dodecane + oxygen + various diluents at an overall stoichiometric ratio $(\varphi=1.0)$ for a droplet size of $d_{0}=3 \mu \mathrm{m}$ from $T_{0}=350 \mathrm{~K}, P_{0}=1$ bar.

Figure 14 - Gas-phase temperatures at the droplet surface and at the 'far-field' as a function of nondimensional surface area for $n$-dodecane + oxygen + various diluents at an overall stoichiometric ratio $(\varphi$ $=1.0$ ) for droplet sizes of $d_{0}=3$ and $8 \mu \mathrm{m}$.

Figure 15 - Gas-phase temperatures at the droplet surface and at the 'far-field' as a function of instantaneous compression ratio for $n$-dodecane + oxygen + various diluents at an overall stoichiometric ratio $(\varphi=1.0)$ for droplet sizes of $d_{0}=3$ and $8 \mu \mathrm{m}$. 
Figure A01 - Normalized sensitivity coefficients of various fuel and diluent gas properties for $n$ dodecane + oxygen + nitrogen at an overall stoichiometric ratio $(\varphi=1.0)$ for droplet sizes of $d_{0}=3$ and $8 \mu \mathrm{m}$.

Figure S1 - Comparison of calculated temperature from an RCEM experiment with predictions from a chemical kinetics model for a pre-mixed charge of hydrogen + 'air' at $\varphi=0.31$ with $T_{0}=297 \mathrm{~K}$ and $P_{0}=$ 1bar. The piston compression time is $14.4 \mathrm{~ms}$ and the pressure at ignition is $P_{i g n} \sim 100 \mathrm{bar}$. Results for a 0D homogeneous reactor model using four detailed chemical kinetic mechanisms are shown.

Figure S2 - Comparison of calculated temperature from an RCEM experiment with predictions from a chemical kinetics model for a pre-mixed charge of $n$-dodecane + 'air' at $\varphi=0.17$ with $T_{0}=350 \mathrm{~K}$ and $P_{0}$ $=1.0$ bar. The piston compression time is $15.7 \mathrm{~ms}$. Results for a $0 \mathrm{D}$ homogeneous reactor model using the detailed mechanism of Westbrook et al. [54] are shown. 


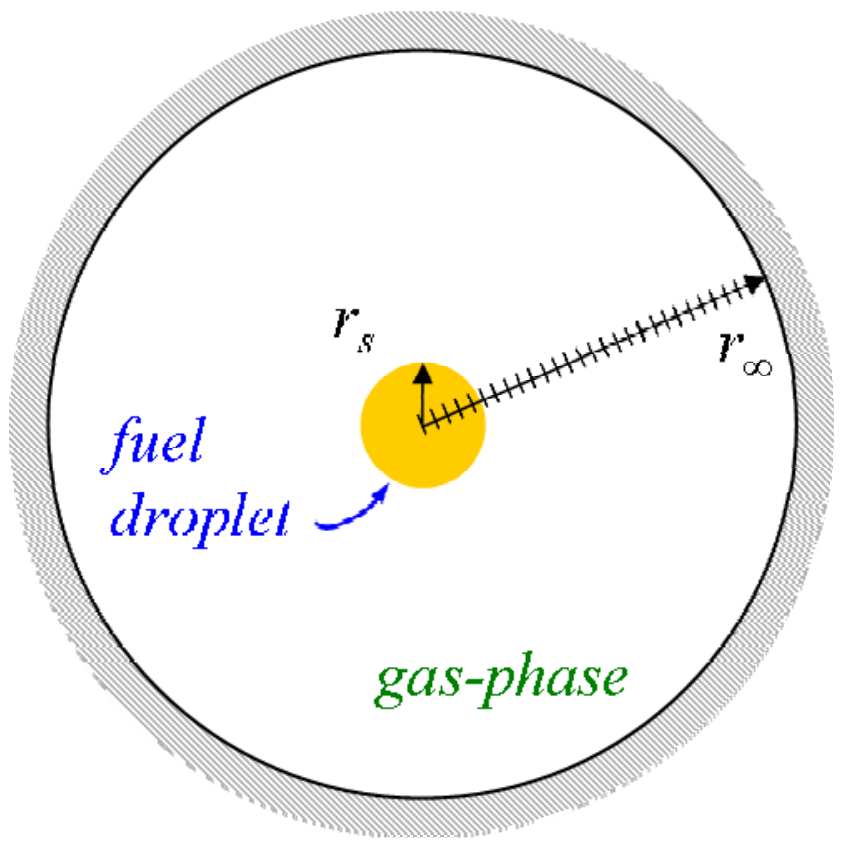

Figure 1 - Schematic of wet compression model illustrating liquid- and gas-phase domains and the location of the 'far-field' boundary. 


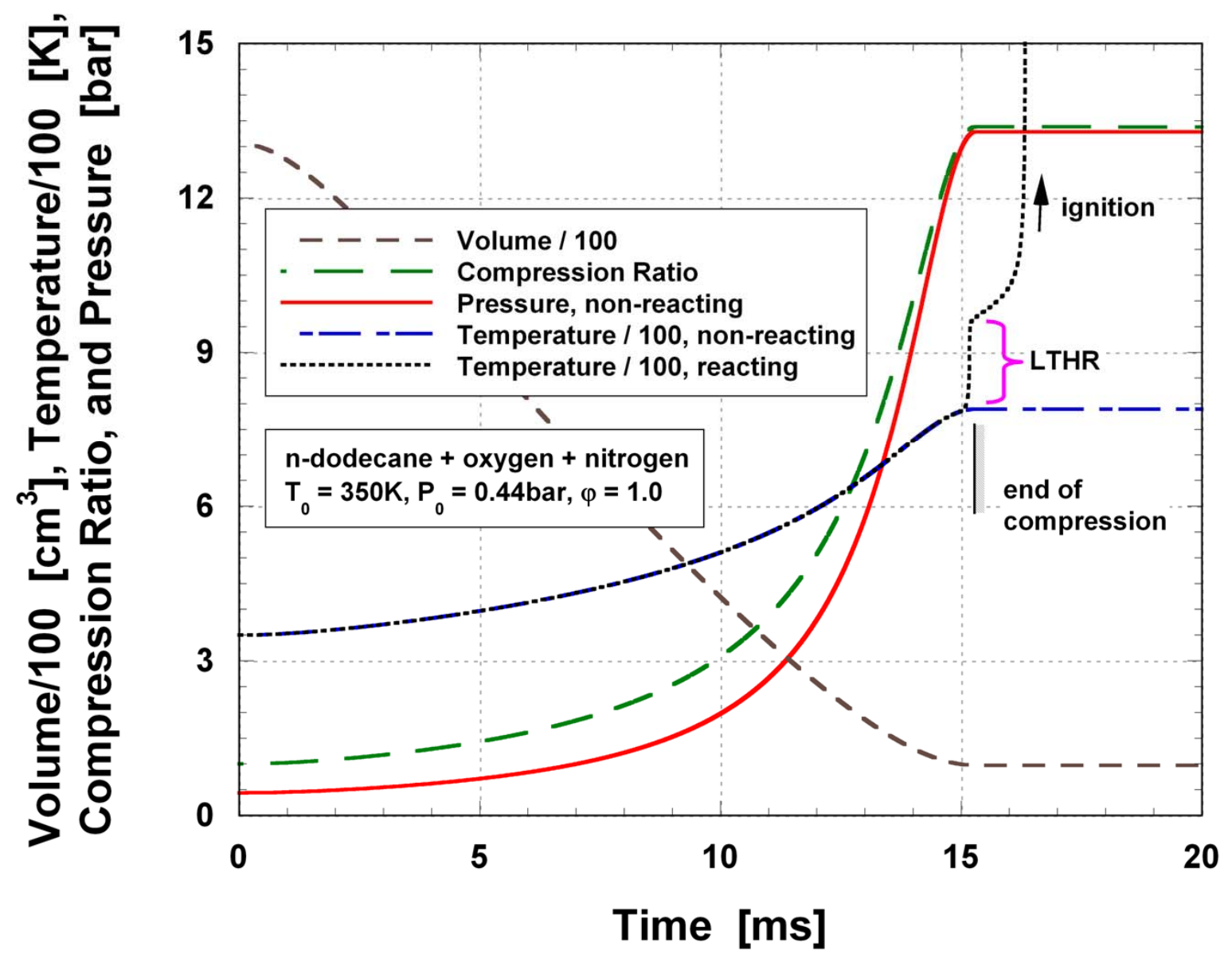

Figure 2 - Representative volume, compression ratio, temperature and pressure histories used in this study where the volume data is typical of rapid compression machines described in the literature. The temperature and pressure trajectories are computed assuming an adiabatic compression process from $T_{0}=$ $350 \mathrm{~K}$ and $P_{0}=0.44 \mathrm{bar}$, respectively, with $\varphi=1.0$ using a fully pre-vaporized / pre-mixed charge. Nonreacting and reacting temperature profiles are shown where the reacting case is computed based on the detailed kinetic mechanism of Westbrook et al. [54]. 


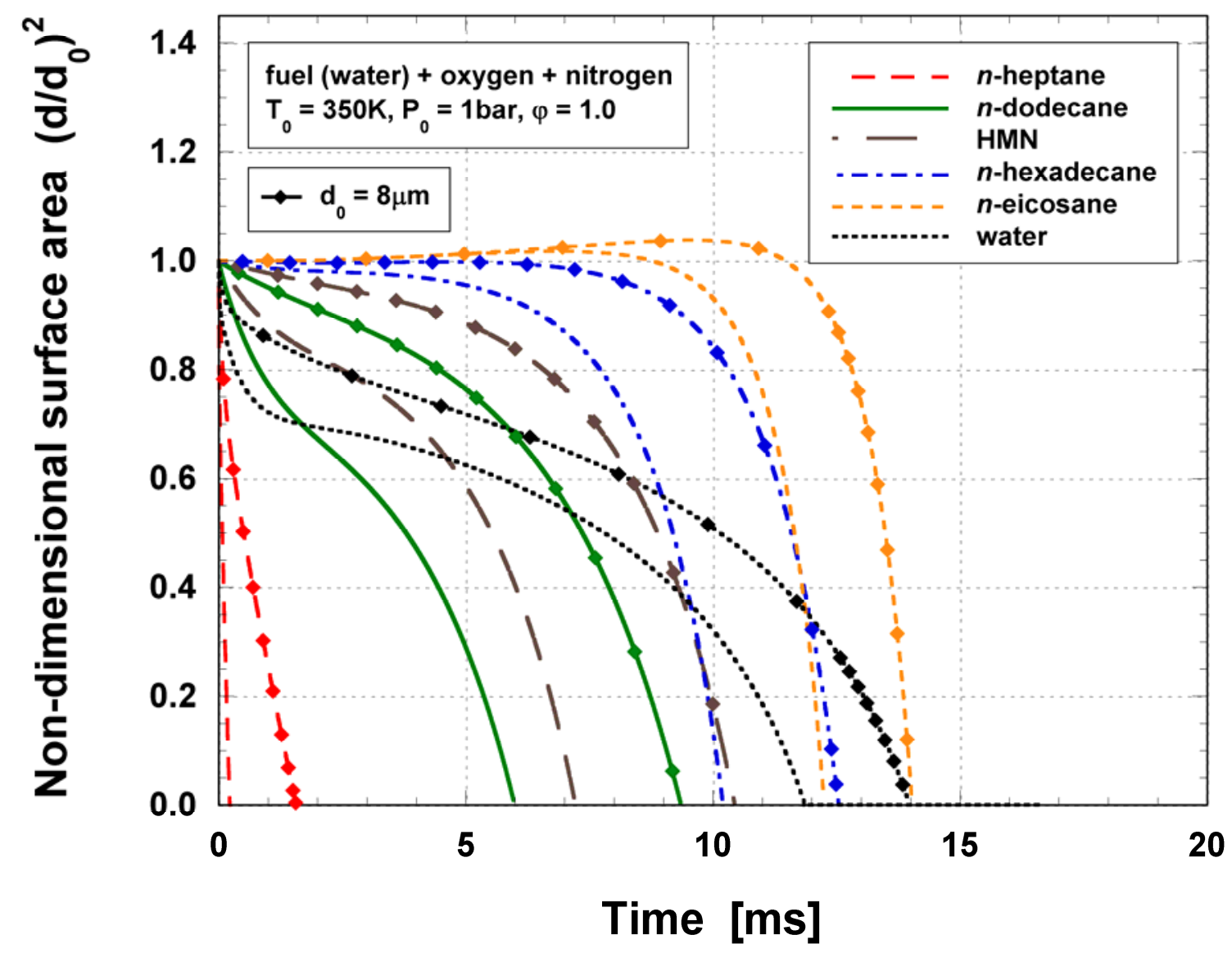

Figure 3 - Non-dimensional droplet surface areas as a function of time for various fuels and water in oxygen + nitrogen at an overall stoichiometric ratio $(\varphi=1.0)$ for droplet sizes of $d_{0}=3$ and $8 \mu \mathrm{m}$. 


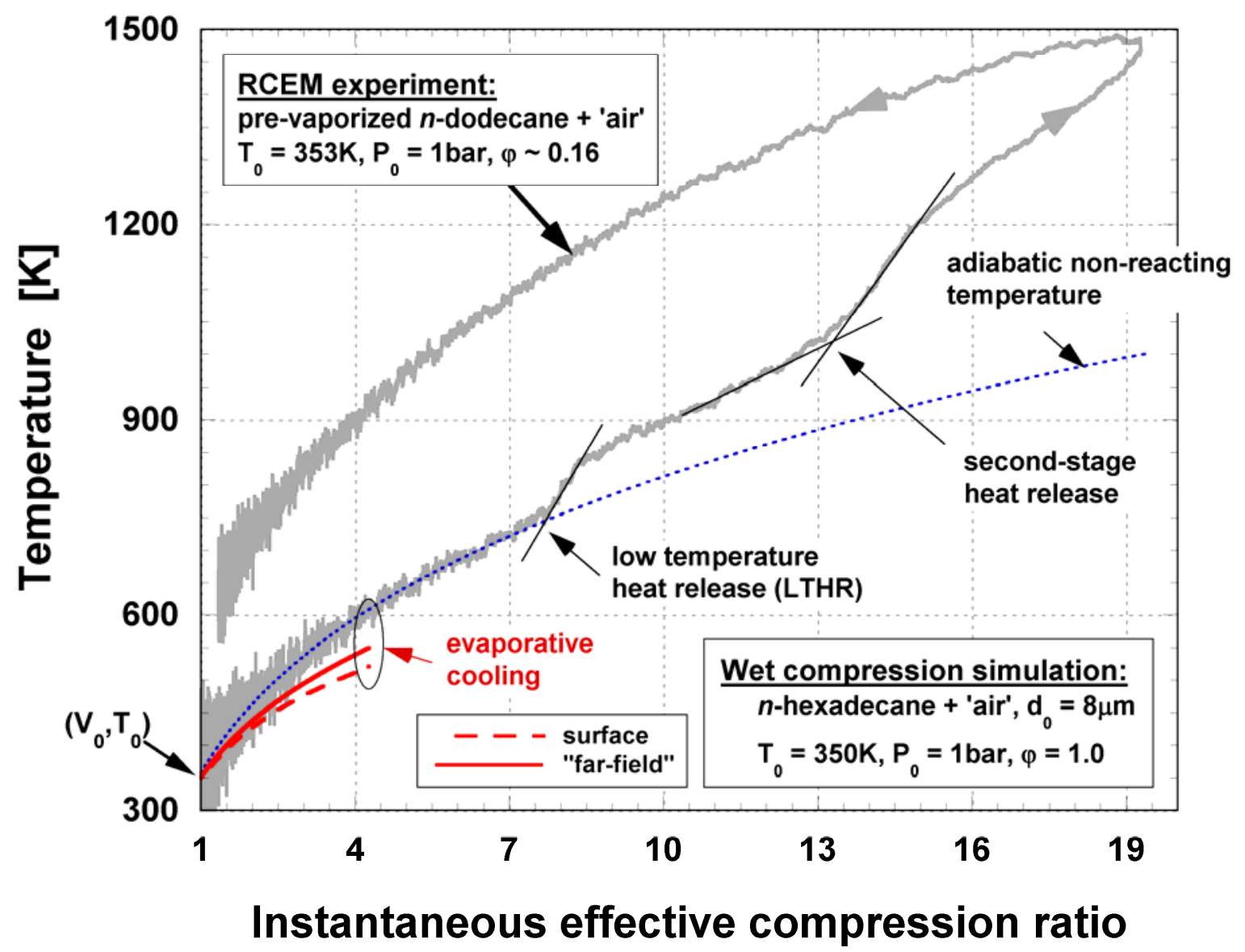

Figure 4 - Comparison of RCEM adiabatic gas temperature for a pre-vaporized $n$-dodecane + 'air' mixture with simulation results of the wet compression model using an unvaporized $n$-hexadecane + 'air' mixture. The initial temperatures for both cases are near $T_{0} \sim 350 \mathrm{~K}$ with initial pressures of $P_{0}=1$ bar. Also indicated here is the adiabatic-core temperature for the $n$-dodecane + 'air' mixture (no heat release). The extent of evaporative cooling that occurs during wet compression at this fuel loading is indicated at $\sim 50-100 \mathrm{~K}$. 


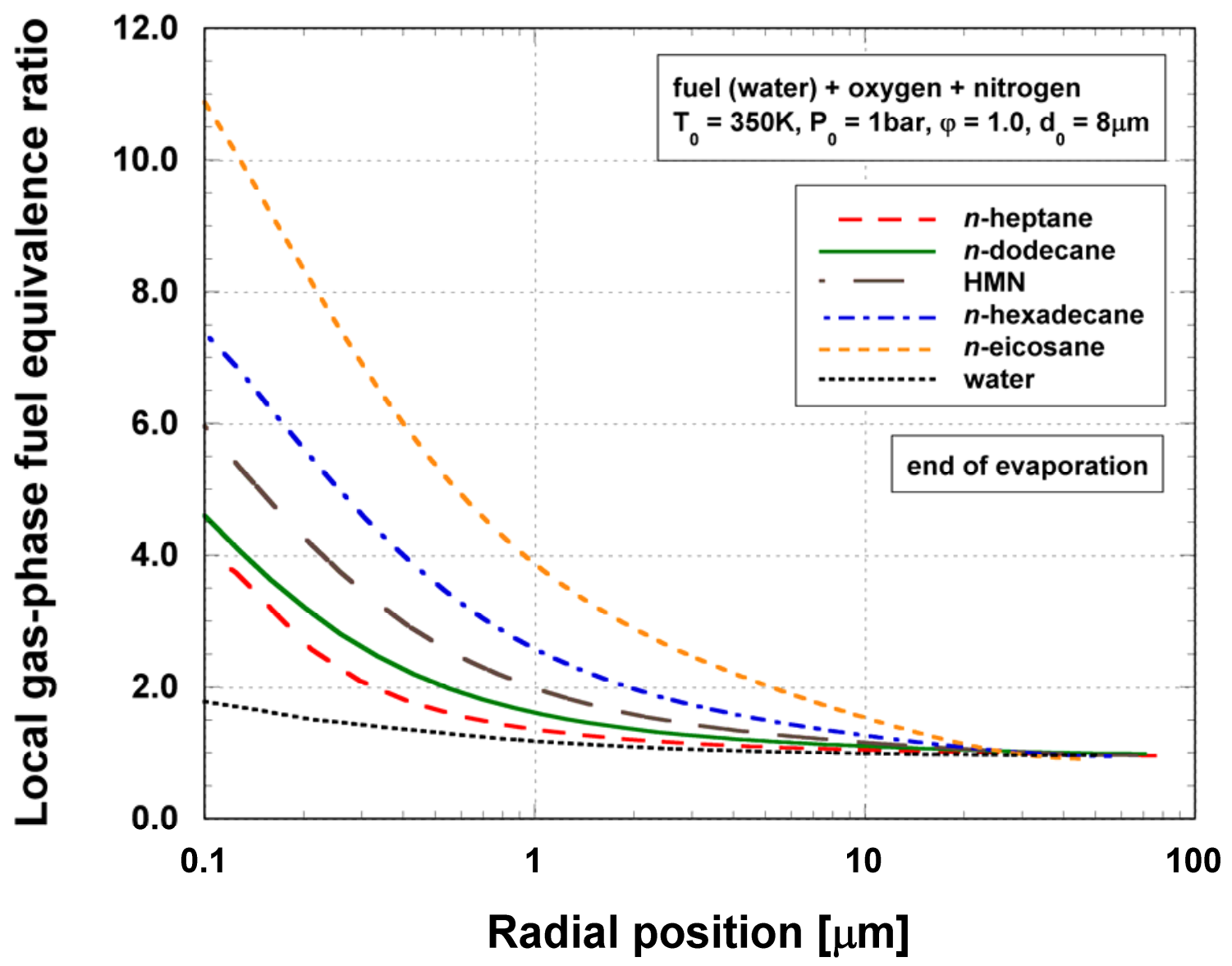

Figure 5a-Gas-phase fuel equivalence ratios and normalized water mass fraction at the end of evaporation as a function of radial position for various fuels and water in oxygen + nitrogen at an overall stoichiometric ratio $(\varphi=1.0)$ for a droplet size of $d_{0}=8 \mu \mathrm{m}$. 


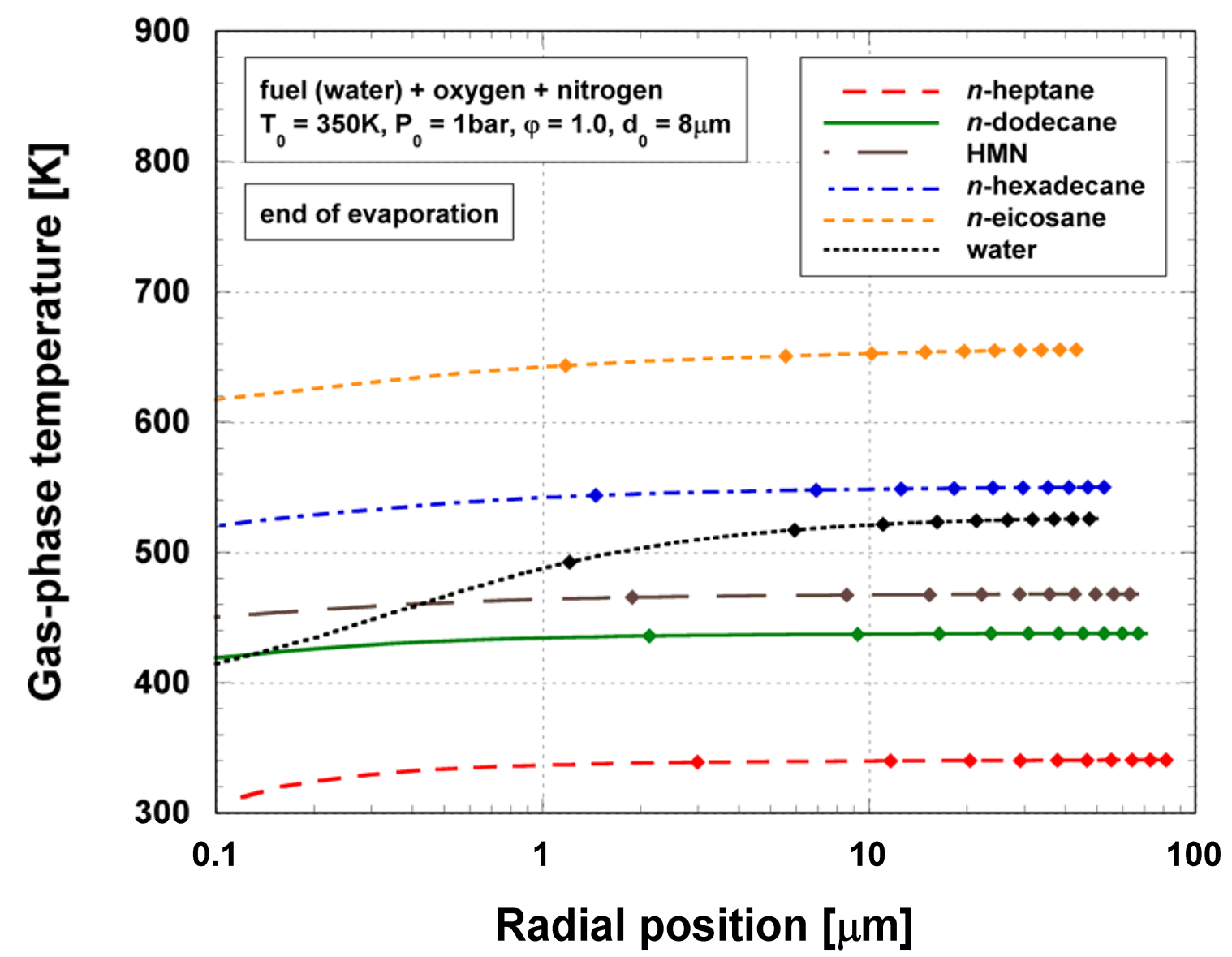

Figure $5 \mathbf{b}$ - Gas-phase temperatures as a function of radial position at the end of evaporation for various fuels and water in oxygen + nitrogen at an overall stoichiometric ratio $(\varphi=1.0)$ for a droplet size of $d_{0}=$ $8 \mu \mathrm{m}$. 


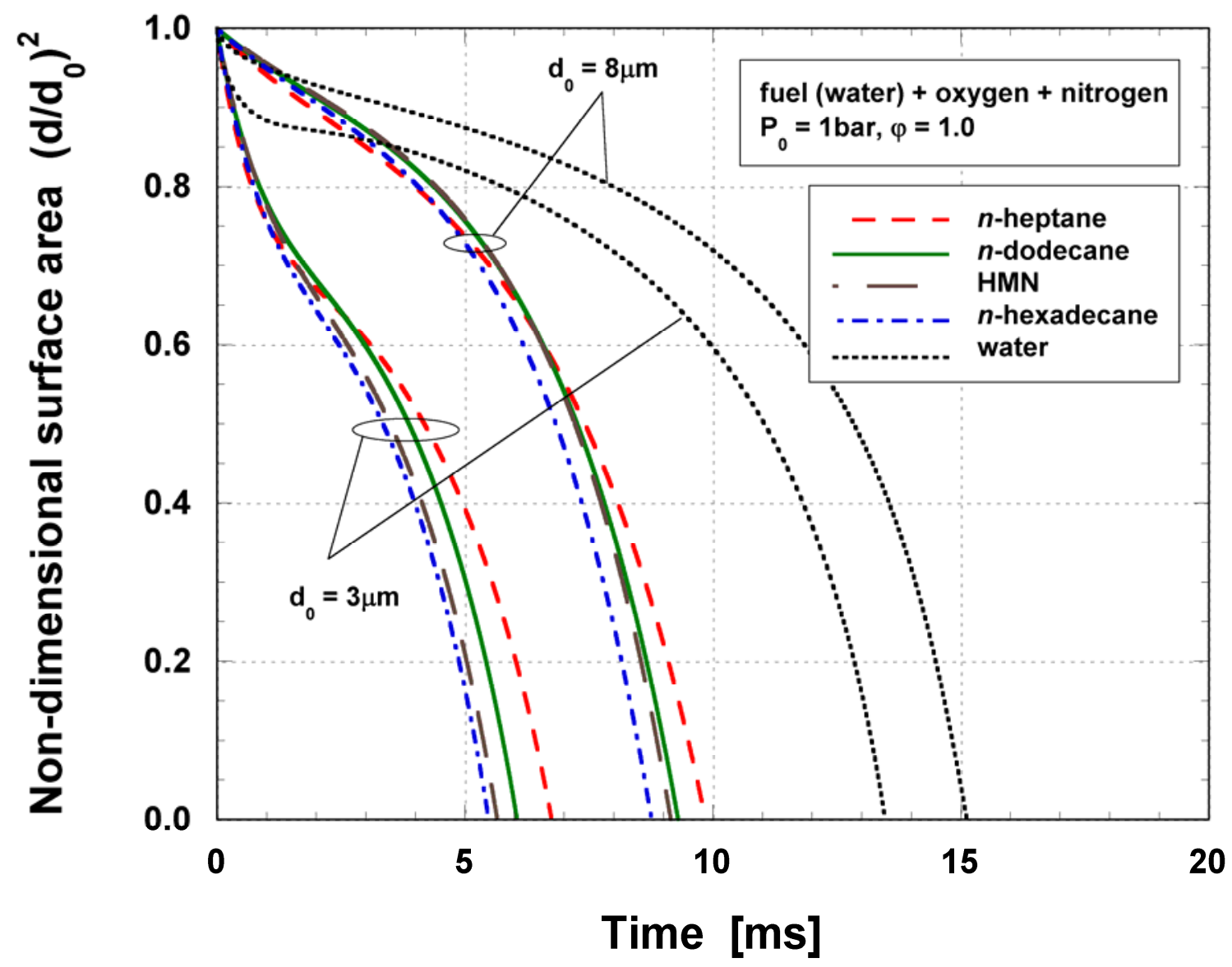

Figure 6 - Non-dimensional droplet surface areas as a function of time for various fuels and water in oxygen + nitrogen at an overall stoichiometric ratio $(\varphi=1.0)$ for droplet sizes of $d_{0}=3$ and $8 \mu \mathrm{m}$ from the initial conditions listed in Table 1, where different initial temperatures are used. 


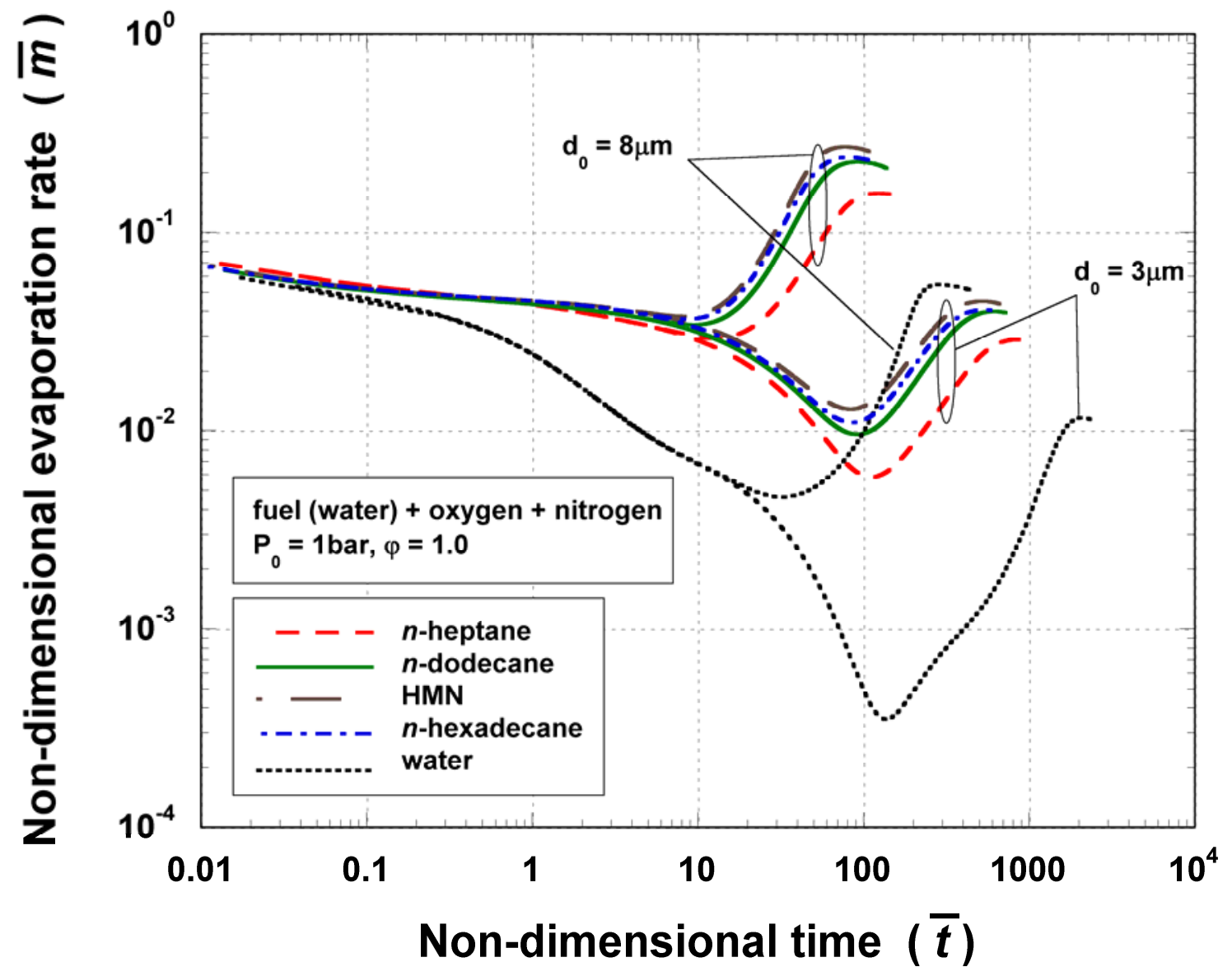

Figure 7 - Non-dimensional evaporation rates as a function of non-dimensional time for various fuels and water in oxygen + nitrogen at an overall stoichiometric ratio $(\varphi=1.0)$ for droplet sizes of $d_{0}=3$ and $8 \mu \mathrm{m}$ from the initial conditions listed in Table 1, where different initial temperatures are used. 


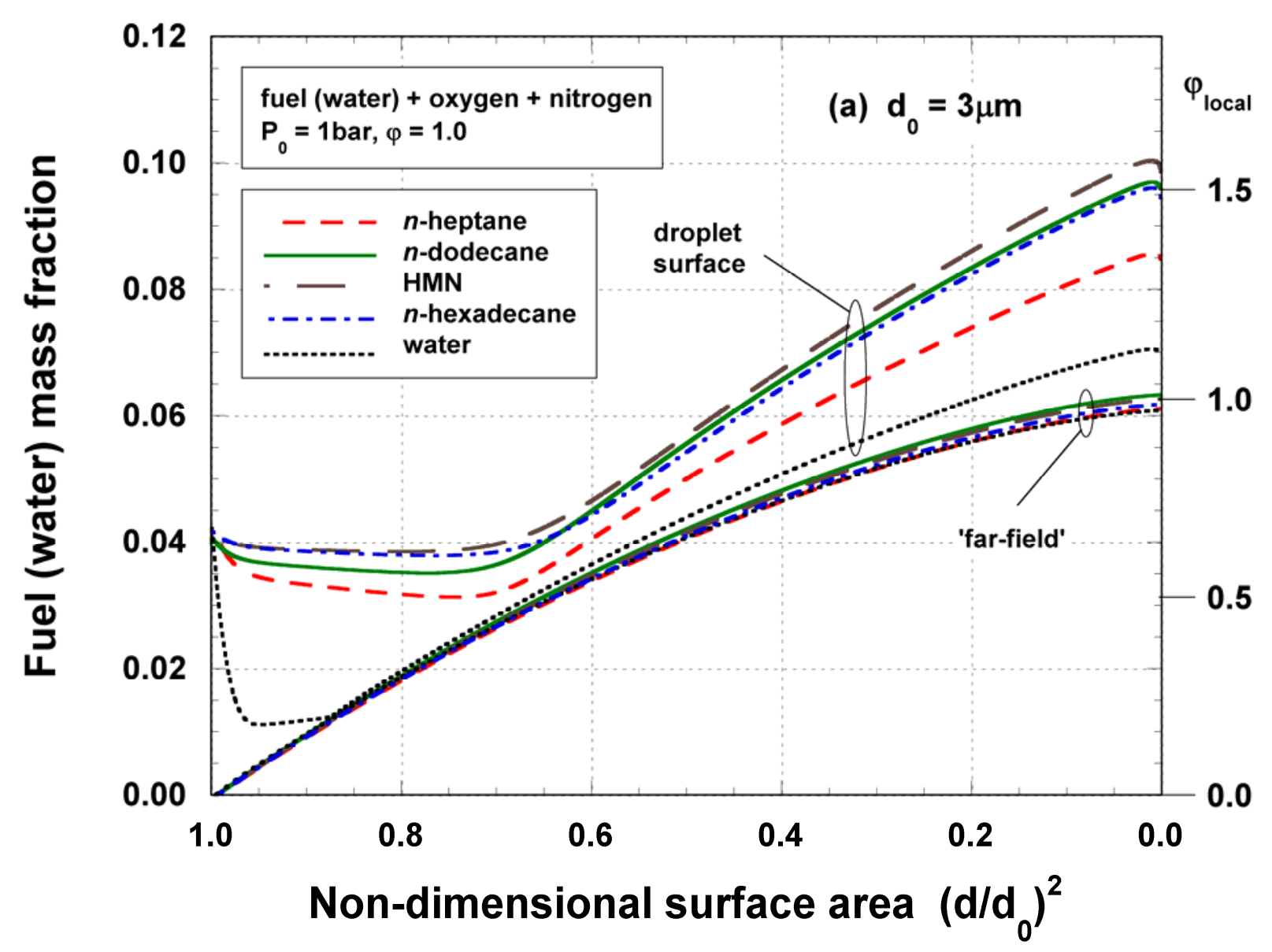

Figure 8 - Gas-phase mass fractions of fuel (water) at the droplet surface and at the 'far-field' as a function of non-dimensional surface area for various fuels and water in oxygen + nitrogen at an overall stoichiometric ratio $(\varphi=1.0)$ for droplet sizes of $d_{0}=3$ and $8 \mu \mathrm{m}$ from the initial conditions listed in Table 1 , where different initial temperatures are used. 


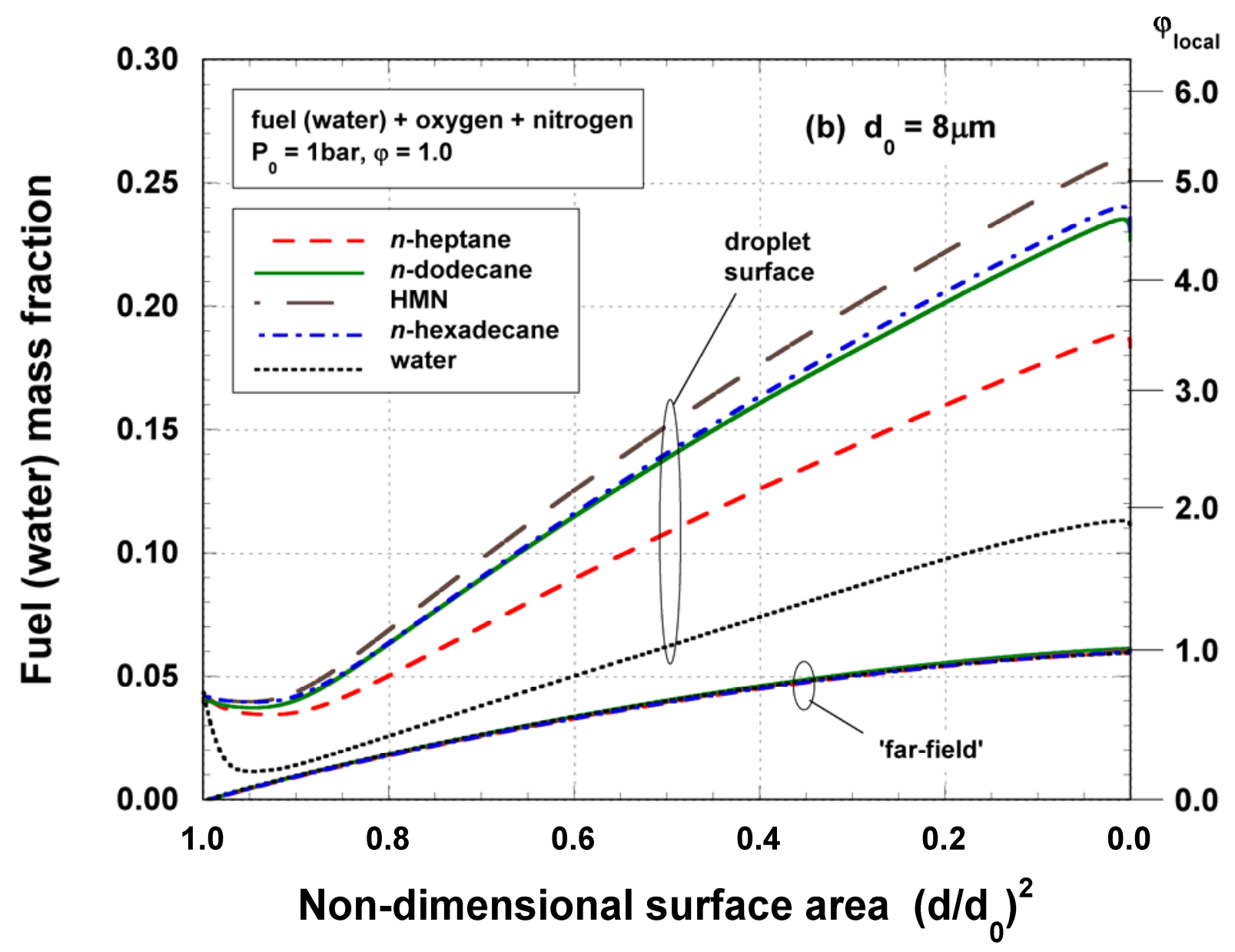




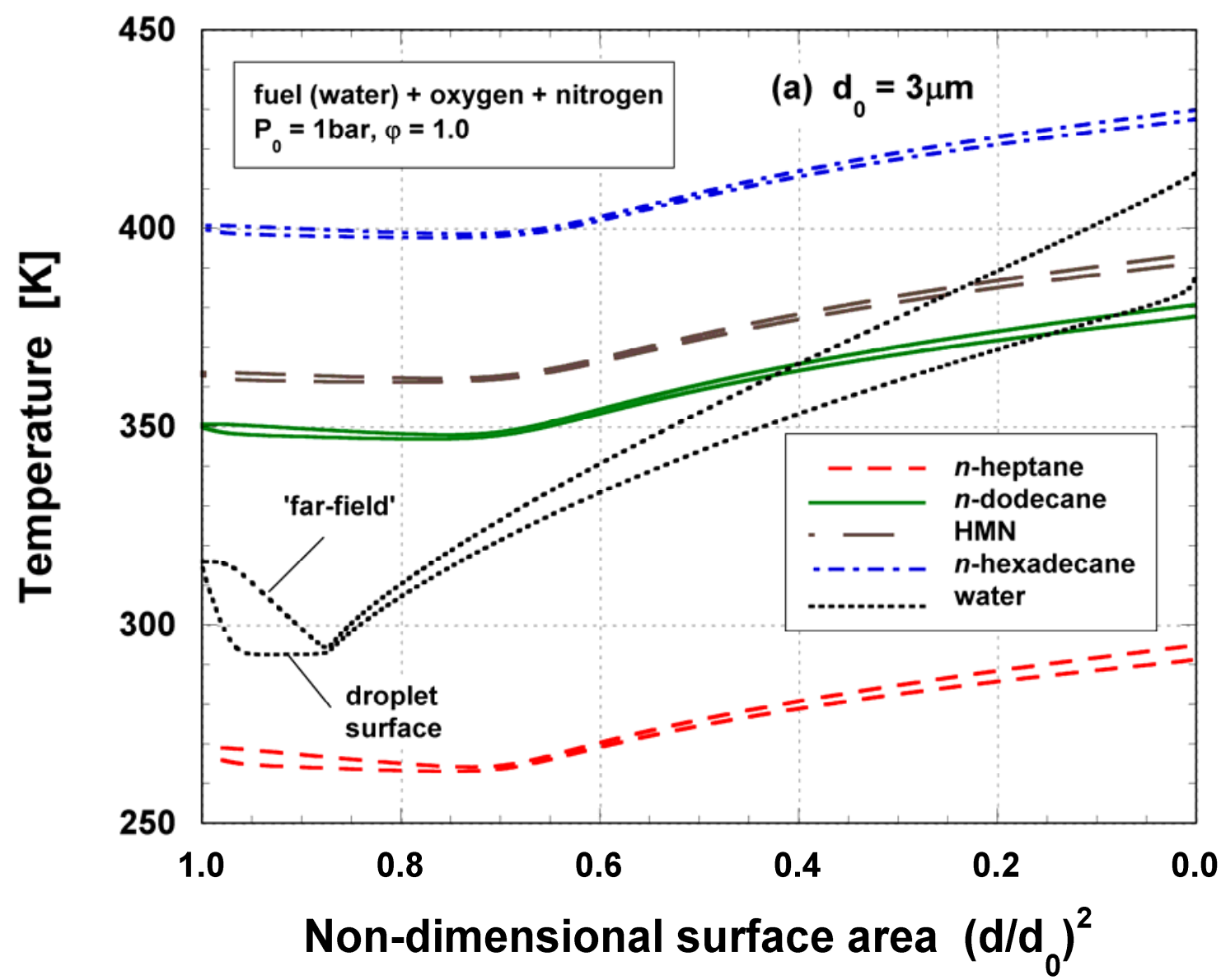

Figure 9 - Gas-phase temperatures at the droplet surface and at the 'far-field' as a function of nondimensional surface area for various fuels and water in oxygen + nitrogen at an overall stoichiometric ratio $(\varphi=1.0)$ for droplet sizes of $d_{0}=3$ and $8 \mu \mathrm{m}$ from the initial conditions listed in Table 1 , where different initial temperatures are used. 


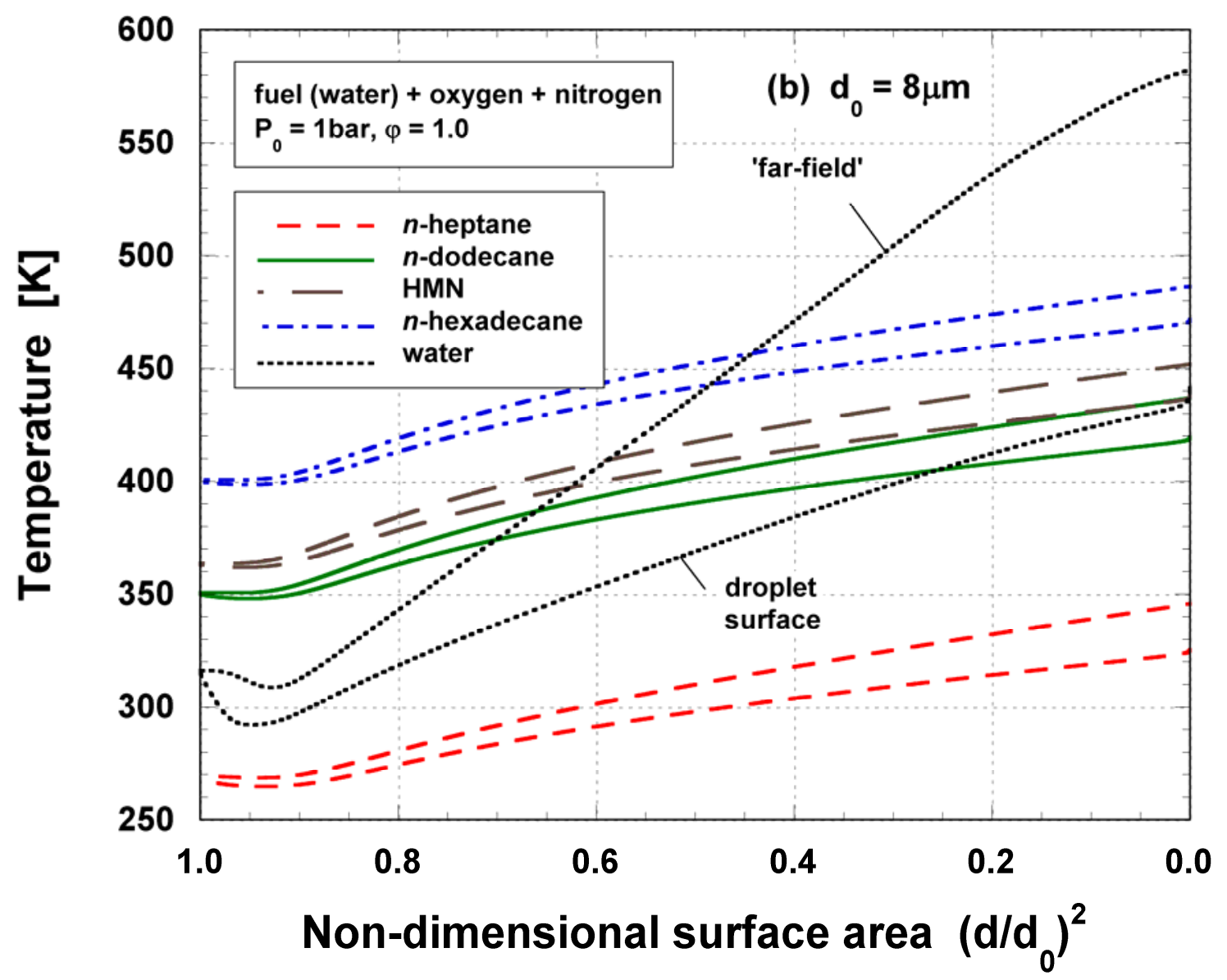




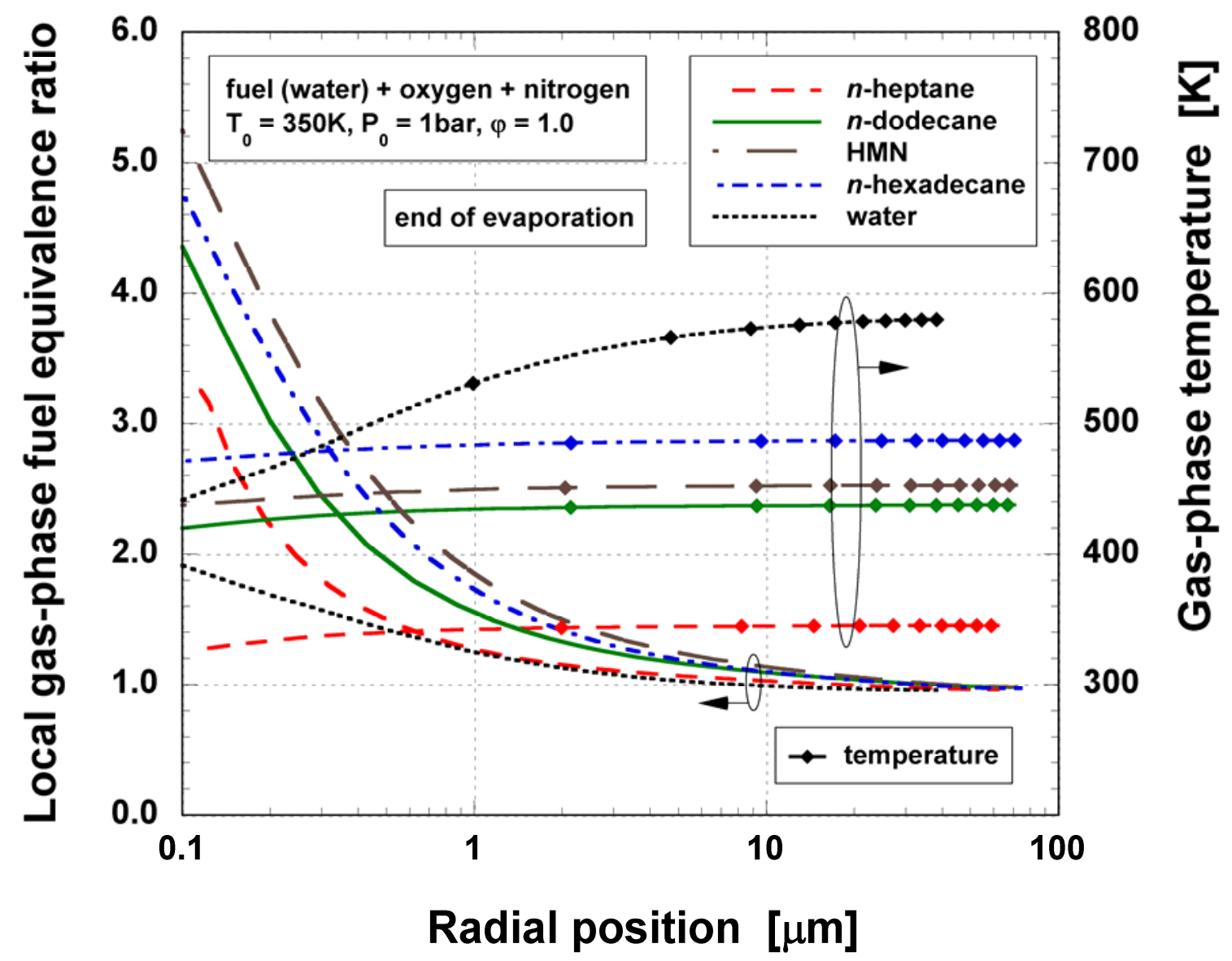

Figure 10 - Gas-phase fuel equivalence ratios and normalized water mass fractions, and temperatures at the end of evaporation as a function of radial position for various fuels and water in oxygen + nitrogen at an overall stoichiometric ratio $(\varphi=1.0)$ for a droplet size of $d_{0}=8 \mu \mathrm{m}$ from the initial conditions listed in Table 1, where different initial temperatures are used. 


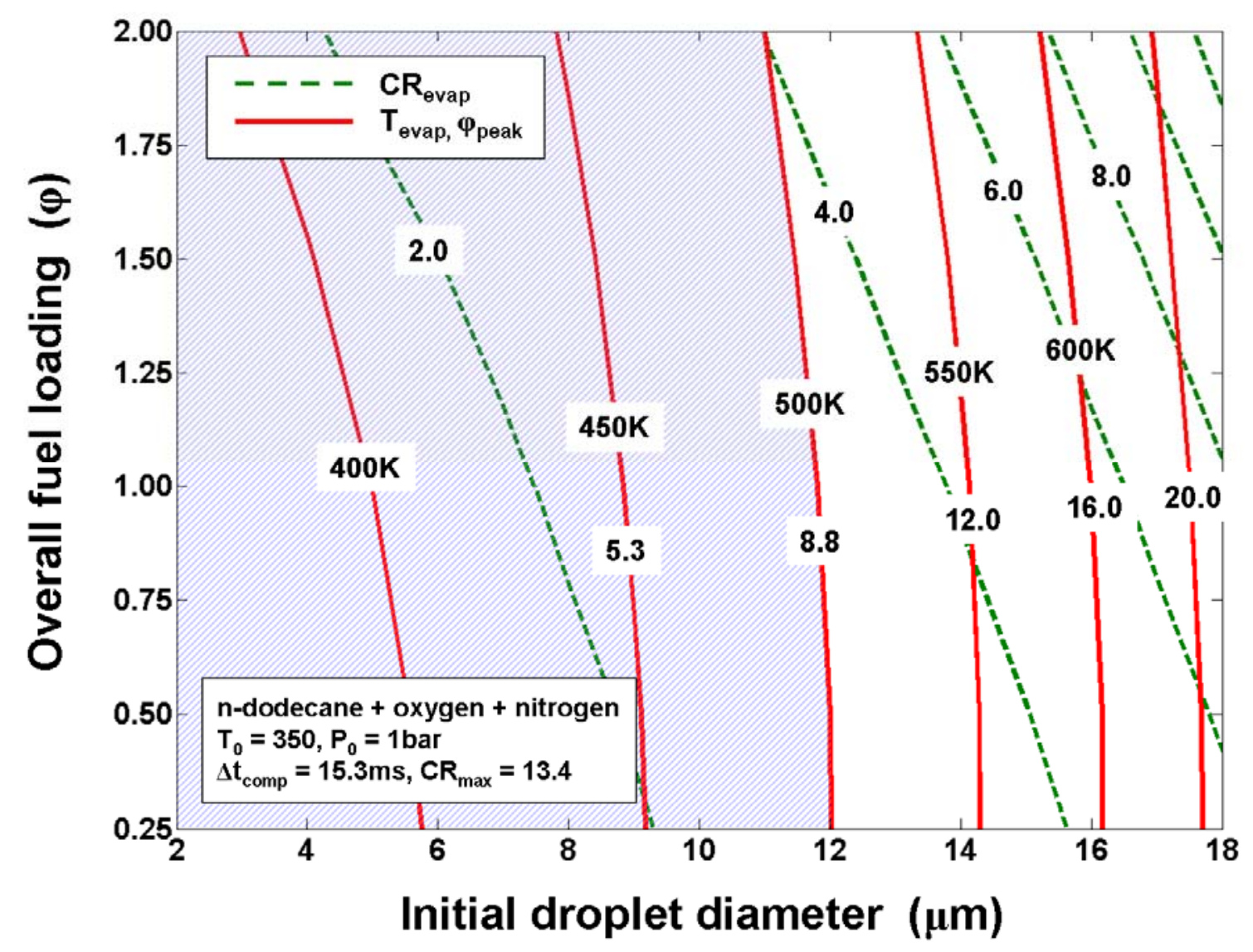

Figure 11 - Predicted 'operating maps' for wet compression of (a) $n$-dodecane + oxygen + nitrogen and (b) $n$-hexadecane + oxygen + nitrogen where the compression ratio, peak gas-phase temperature and peak equivalence ratio at the end of the vaporization process are indicated for a range of initial droplet diameters at various overall fuel loadings. 


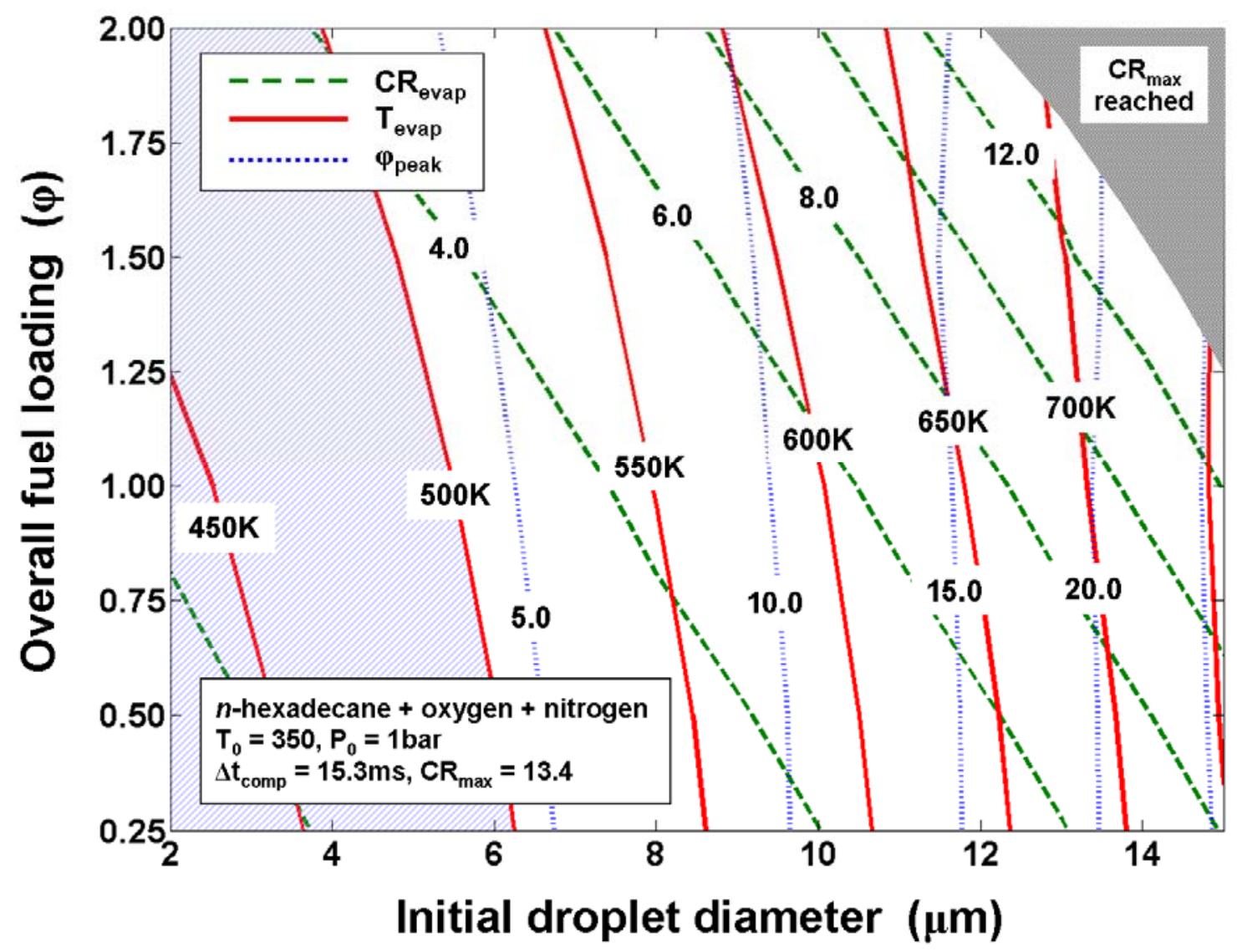




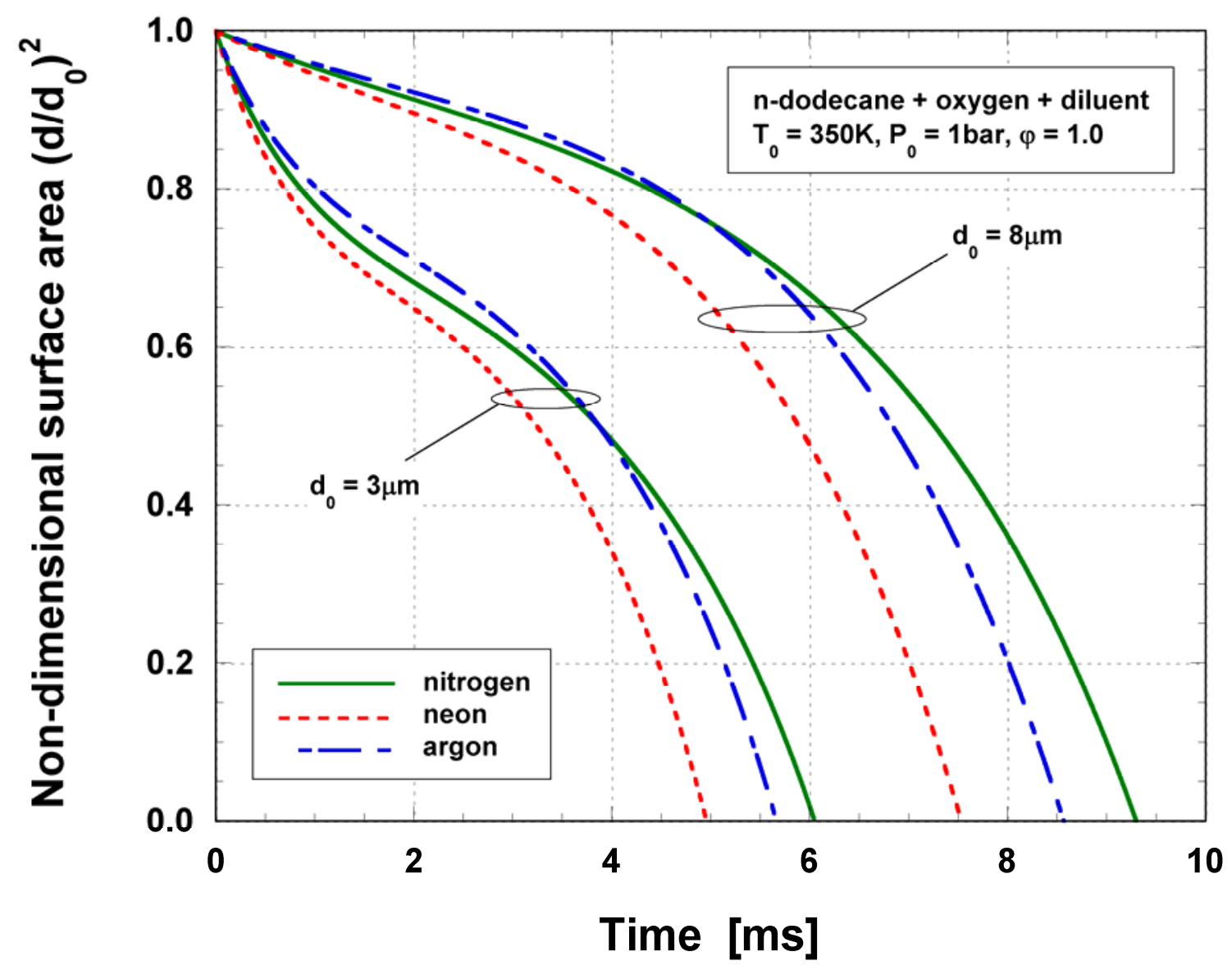

Figure 12 - Non-dimensional droplet surface areas as a function of time for $n$-dodecane + oxygen and various diluents at an overall stoichiometric ratio $(\varphi=1.0)$ for droplet sizes of $d_{0}=3$ and $8 \mu \mathrm{m}$. 


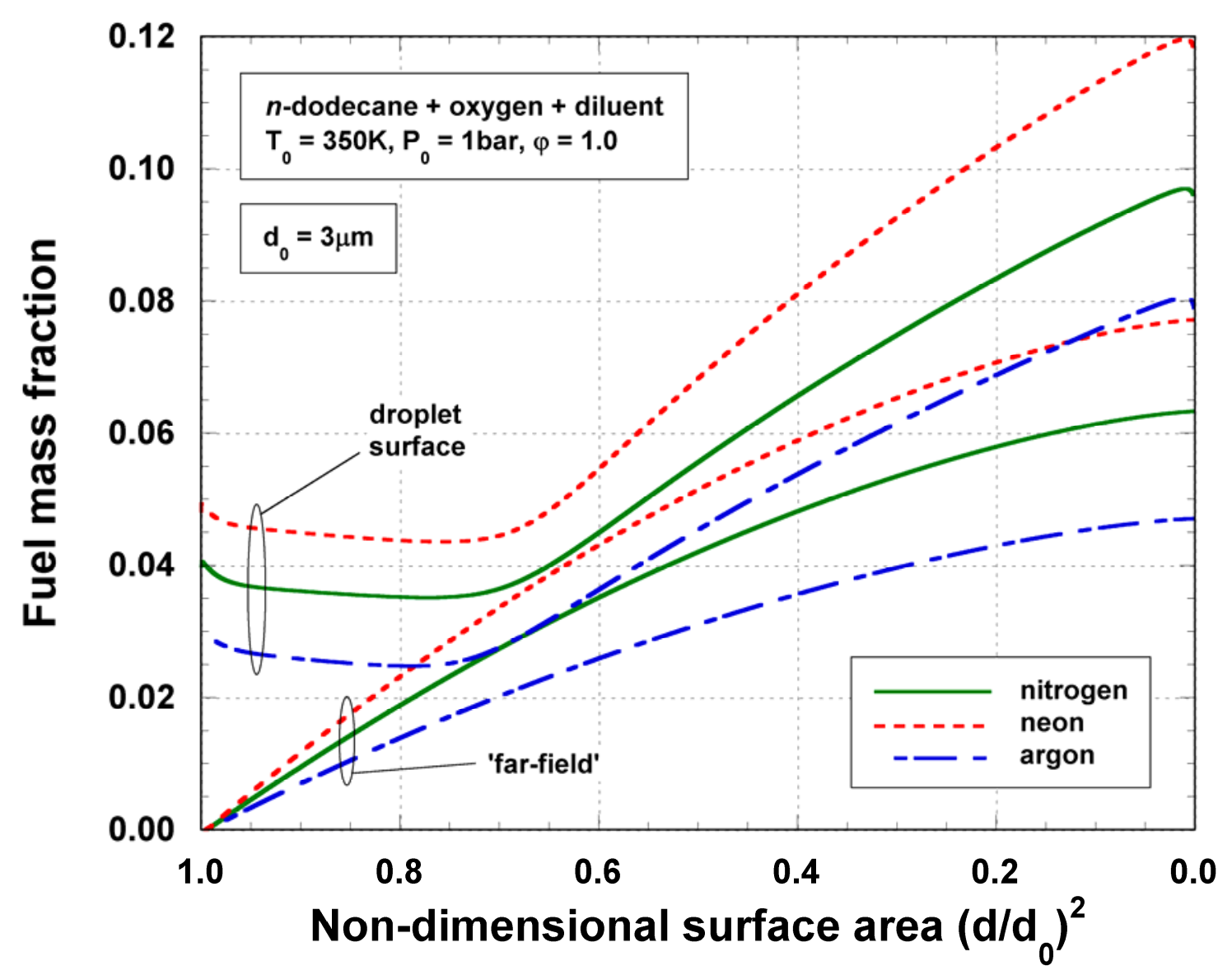

Figure 13 - Gas-phase fuel mass fractions at the droplet surface and at the 'far-field' as a function of non-dimensional surface area for $n$-dodecane + oxygen + various diluents at an overall stoichiometric ratio $(\varphi=1.0)$ for a droplet size of $d_{0}=3 \mu \mathrm{m}$. 


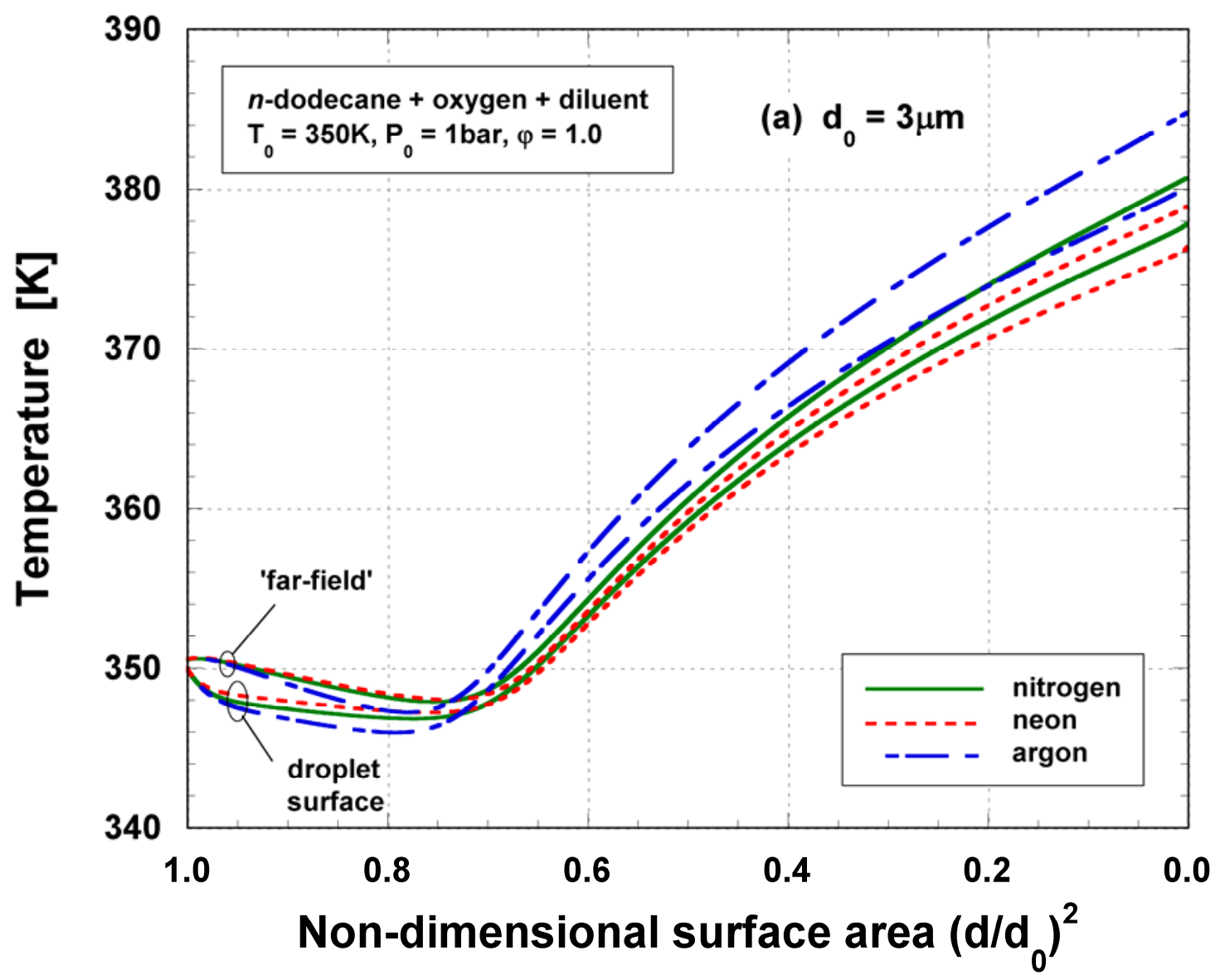

Figure 14 - Gas-phase temperatures at the droplet surface and at the 'far-field' as a function of nondimensional surface area for $n$-dodecane + oxygen + various diluents at an overall stoichiometric ratio $(\varphi$ $=1.0$ ) for droplet sizes of $d_{0}=3$ and $8 \mu \mathrm{m}$. 


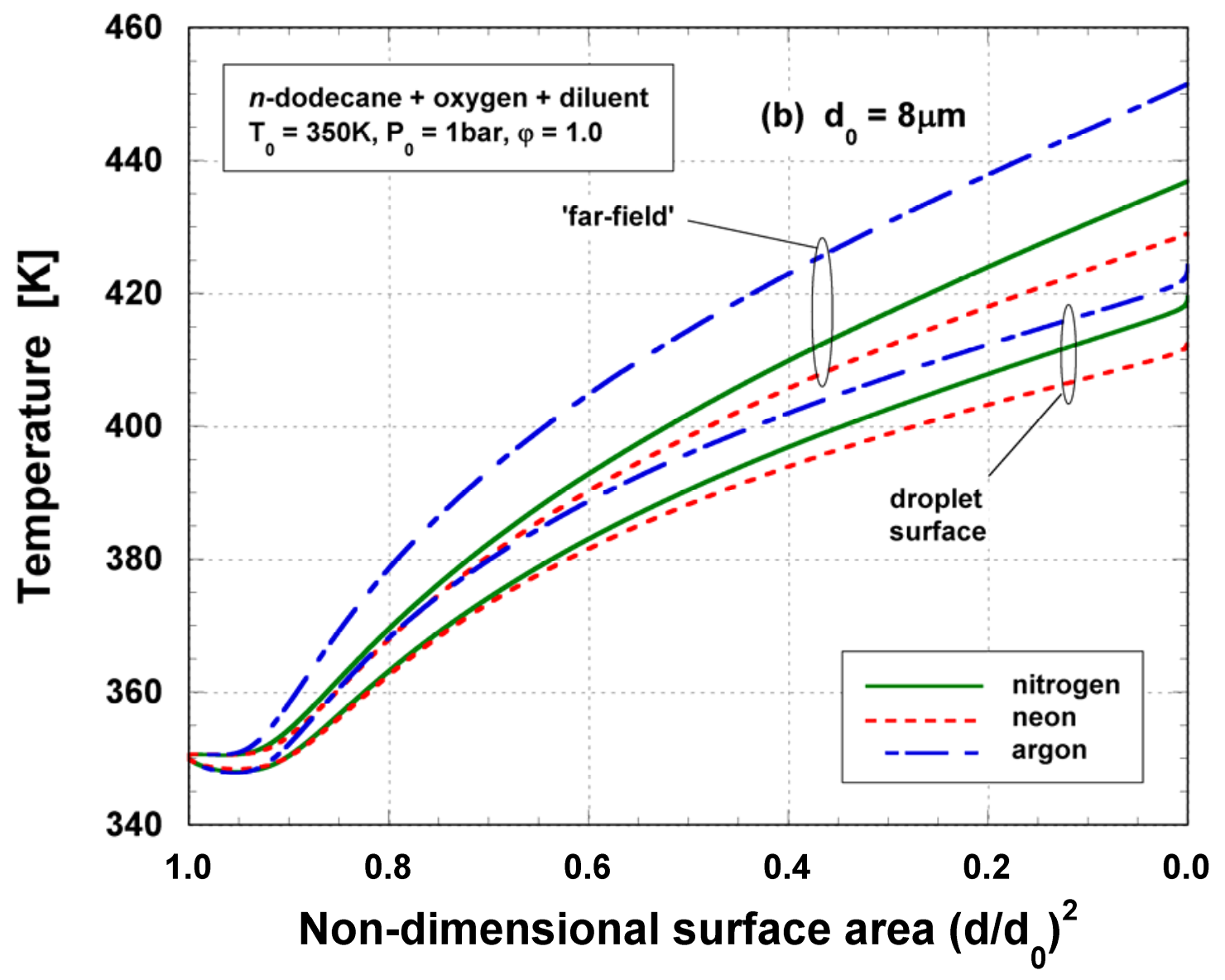




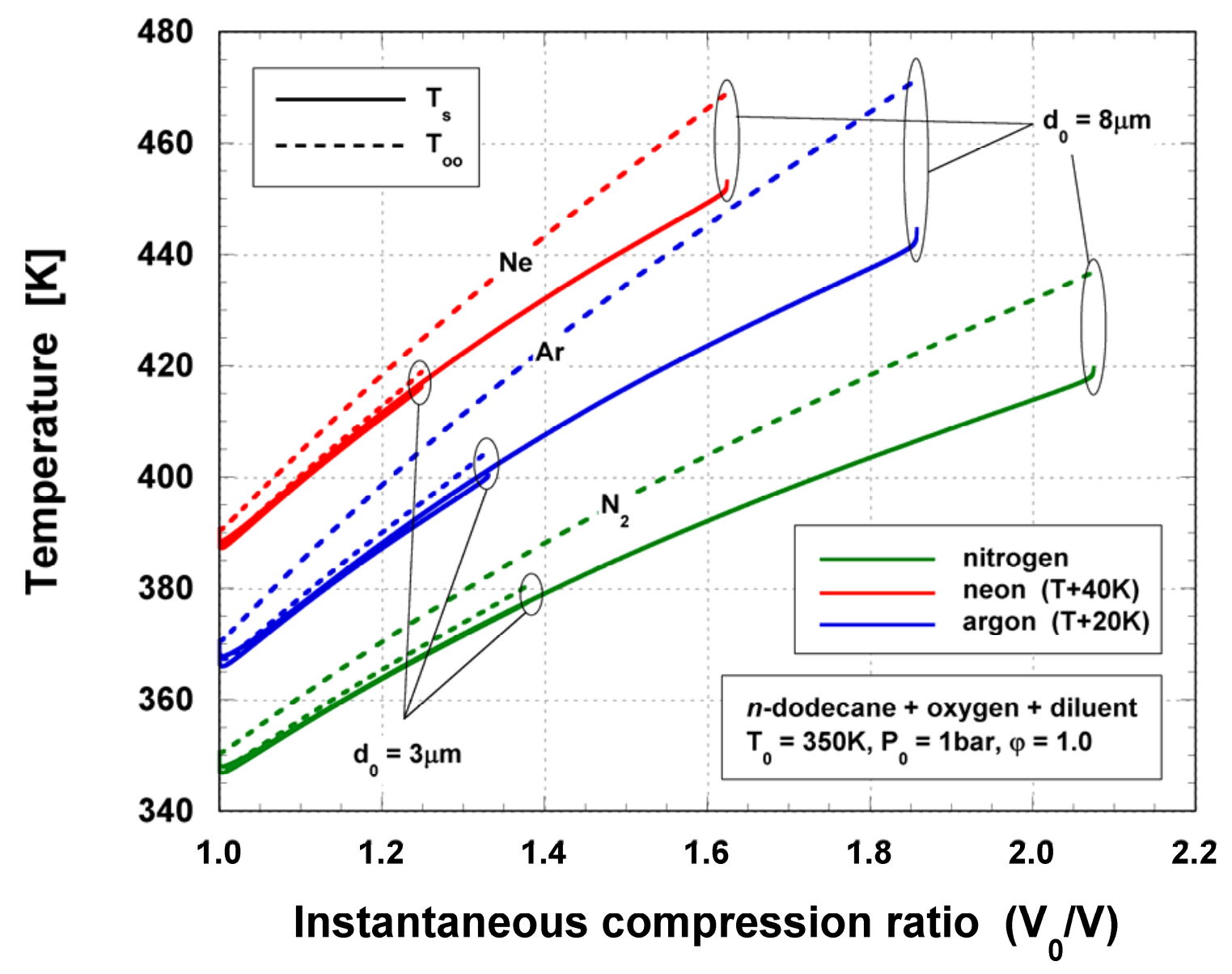

Figure 15 - Gas-phase temperatures at the droplet surface and at the 'far-field' as a function of instantaneous compression ratio for $n$-dodecane + oxygen + various diluents at an overall stoichiometric ratio $(\varphi=1.0)$ for droplet sizes of $d_{0}=3$ and $8 \mu \mathrm{m}$. 


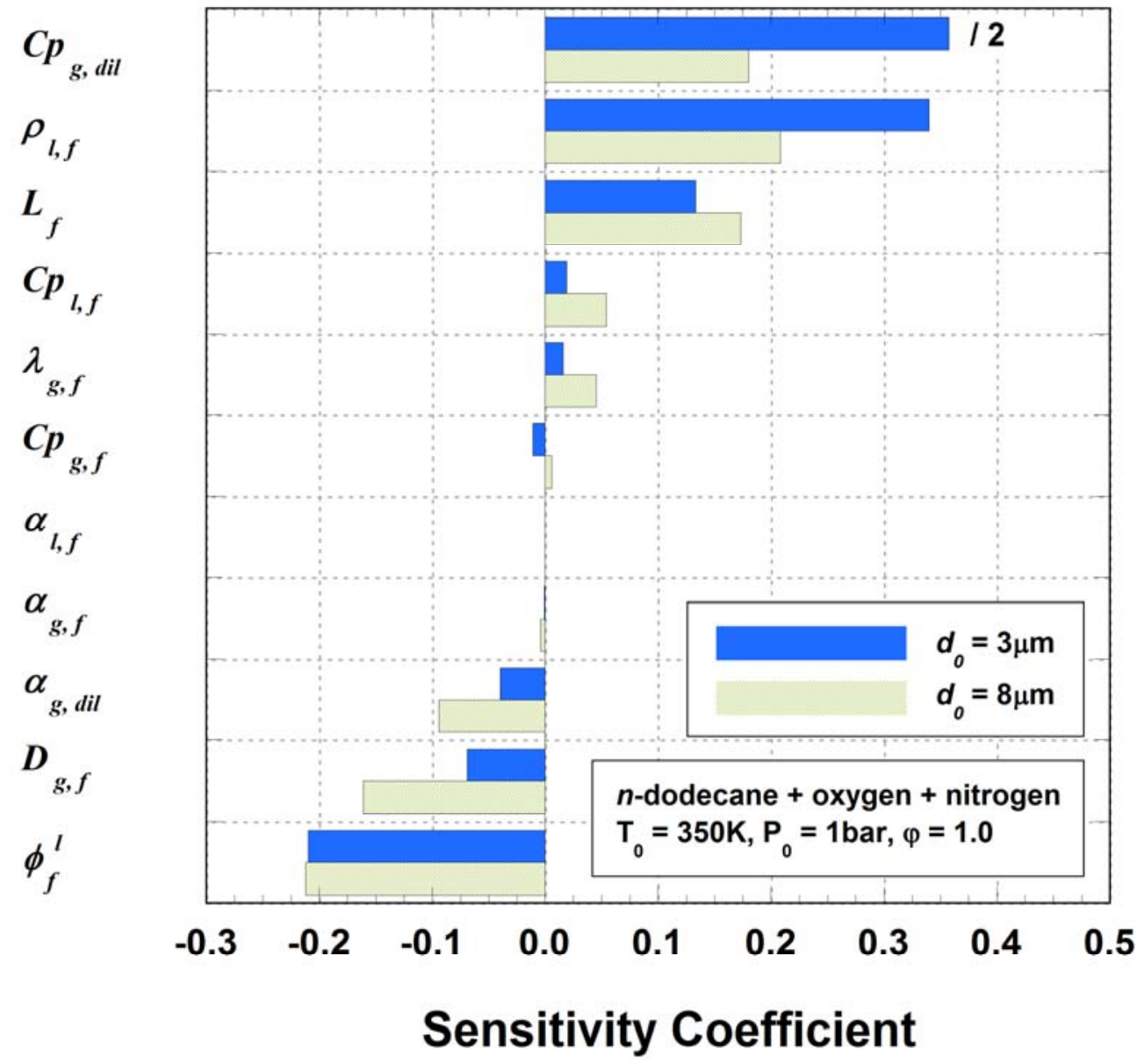

Figure A01 - Normalized sensitivity coefficients of various fuel and diluent gas properties for $n$ dodecane + oxygen + nitrogen at an overall stoichiometric ratio $(\varphi=1.0)$ for droplet sizes of $d_{0}=3$ and $8 \mu \mathrm{m}$. 


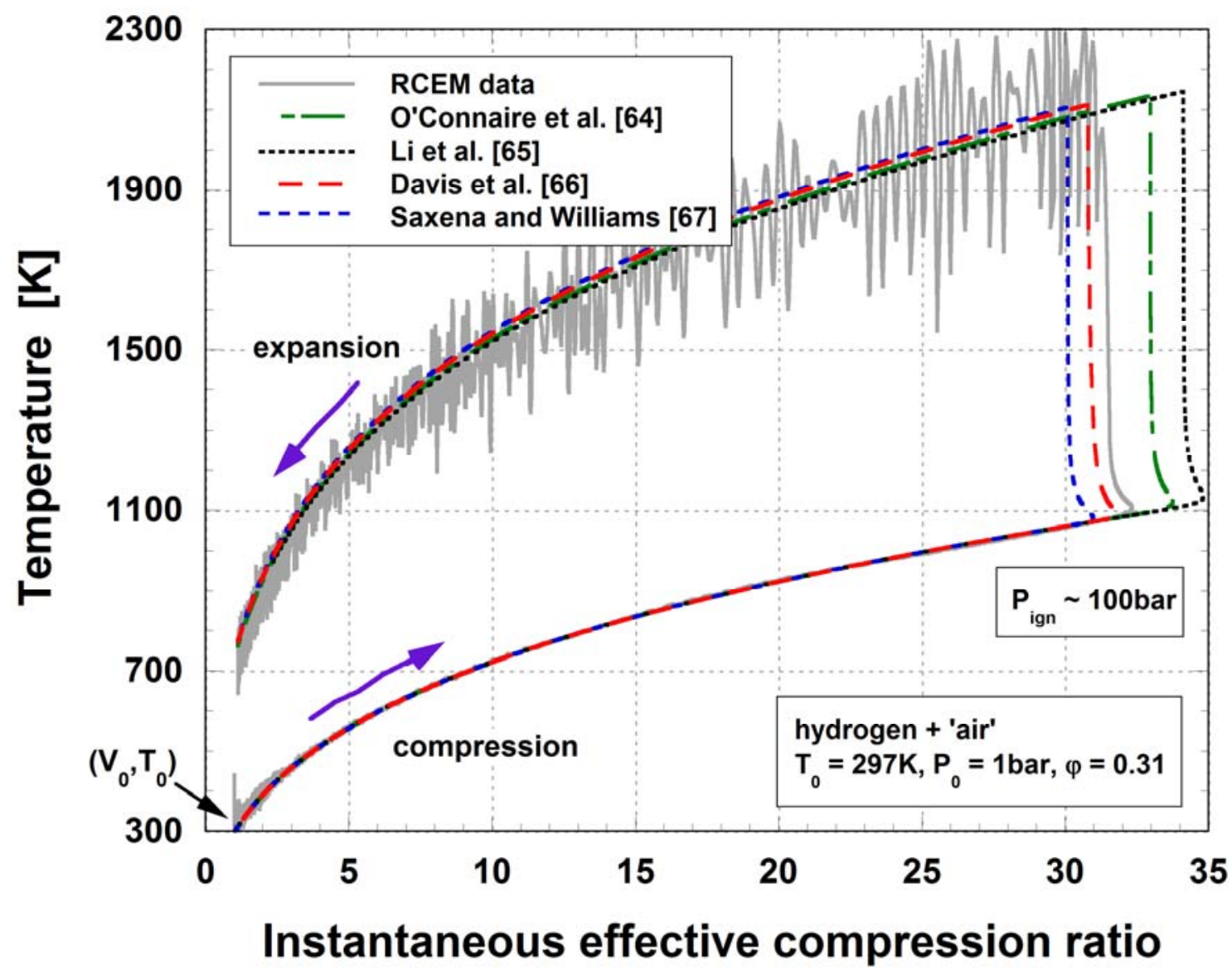

Figure S1 - Comparison of calculated temperature from an RCEM experiment with predictions from a chemical kinetics model for a pre-mixed charge of hydrogen + 'air' at $\varphi=0.31$ with $T_{0}=297 \mathrm{~K}$ and $P_{0}=$ 1 bar. The piston compression time is $14.4 \mathrm{~ms}$ and the pressure at ignition is $P_{\text {ign }} \sim 100 \mathrm{bar}$. Results for a OD homogeneous reactor model using four detailed chemical kinetic mechanisms are shown. 


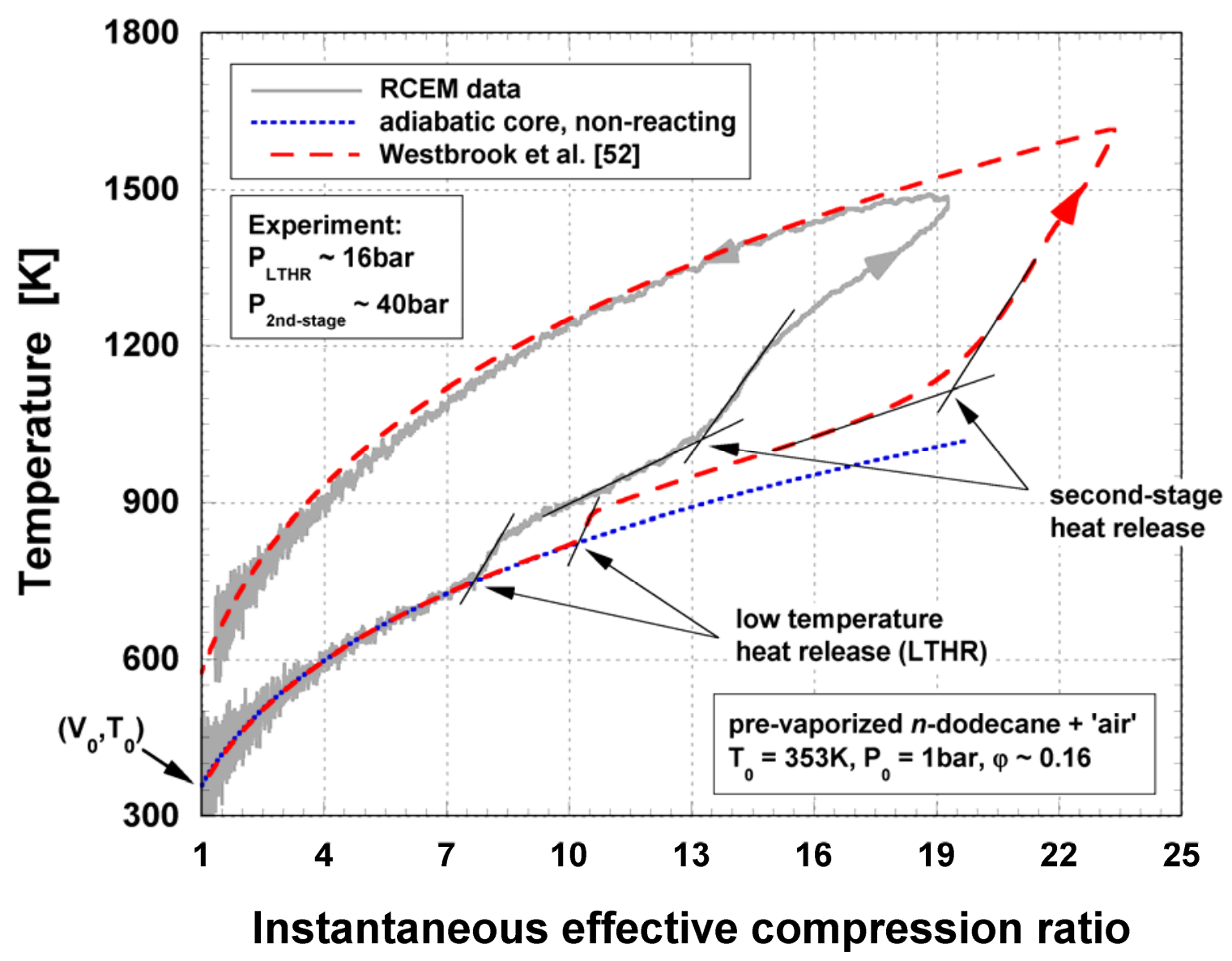

Figure S2 - Comparison of calculated temperature from an RCEM experiment with predictions using a chemical kinetics model for a pre-mixed charge of $n$-dodecane + 'air' at $\varphi=0.17$ with $T_{0}=350 \mathrm{~K}$ and $P_{0}$ $=1.0$ bar. The piston compression time is $15.7 \mathrm{~ms}$. Results for a $0 \mathrm{D}$ homogeneous reactor model using the detailed mechanism of Westbrook et al. [54] are shown. 
Table 1 - Properties and initial conditions (for section 3.1.2) for various fuels (and water) in 'air' using an initial pressure of $P_{0}=1$ bar and $\varphi=1.0$; the actual mass ratio of water to 'air' is specified.

\begin{tabular}{|c|c|c|c|c|c|c|}
\hline & $n$-heptane & $n$-dodecane & $\mathrm{HMN}$ & $n$-hexadecane & $n$-eicosane & water \\
\hline Chemical formula & $\mathrm{C}_{7} \mathrm{H}_{16}$ & $\mathrm{C}_{12} \mathrm{H}_{26}$ & $\mathrm{C}_{16} \mathrm{H}_{34}$ & $\mathrm{C}_{16} \mathrm{H}_{34}$ & $\mathrm{C}_{20} \mathrm{H}_{42}$ & $\mathrm{H}_{2} \mathrm{O}$ \\
$M_{f}(\mathrm{~g} / \mathrm{mol})$ & 100.21 & 170.34 & 226.44 & 226.44 & 282.55 & 18.01 \\
$\left(m_{f} / m_{a}\right)_{\text {stoich }}$ & 0.066 & 0.067 & 0.067 & 0.067 & 0.067 & $0.066^{\mathrm{A}}$ \\
$P^{\text {sat }} @ 300 \mathrm{~K}(\mathrm{Torr})^{\mathrm{a}}$ & 45.1 & 0.135 & 0.044 & 0.002 & $2 \times 10^{-5}$ & 23.8 \\
$T_{\text {boil }} @ 1 \mathrm{~atm}(\mathrm{~K})^{\mathrm{a}}$ & 372 & 489 & 520 & 560 & 616 & 373 \\
$T_{0}(\mathrm{~K})^{\mathrm{a}}$ & 269 & 350 & 363 & 400 & 444 & 315 \\
$\rho_{l, 0}\left(\mathrm{~g} / \mathrm{cm}^{3}\right)^{\mathrm{a}}$ & 0.706 & 0.734 & 0.749 & 0.702 & 0.688 & 0.975 \\
$r_{\infty, 0} / r_{s, 0}$ & 20.4 & 22.5 & 22.8 & 23.2 & 23.8 & 24.0 \\
$L_{f, 0}(\mathrm{~J} / \mathrm{g}-\mathrm{K})^{\mathrm{a}}$ & 375 & 313 & 248 & 288 & 272 & 2,520 \\
$\left(\rho_{g} D_{g}\right)_{s, 0}(\mathrm{cP})^{\mathrm{a}}$ & 0.0083 & 0.0071 & 0.0063 & 0.0068 & 0.0065 & 0.0290 \\
Le $_{s, 0}$ & 2.64 & 3.75 & 4.33 & 4.28 & 4.73 & 0.85 \\
\hline
\end{tabular}

a - based on thermophysical models discussed in section 2.0.

Table 2 - Initial properties for various mixtures of $n$-dodecane + oxygen + diluent at an initial temperature and pressure of $T_{0}=350 \mathrm{~K}$ and $P_{0}=1 \mathrm{bar}$, respectively, with an overall fuel loading of $\varphi=1.0$.

\begin{tabular}{|c|c|c|c|}
\hline & nitrogen & neon & argon \\
\hline$M_{g, 0}(\mathrm{~g} / \mathrm{mol})$ & 28.86 & 22.66 & 38.28 \\
$\left(m_{f} / m_{a}\right)_{\text {stoich }}$ & 0.067 & 0.086 & 0.050 \\
$r_{\infty, 0} / r_{s, 0}$ & 22.5 & 22.5 & 22.5 \\
$\rho_{g, 0}\left(\mathrm{~g} / \mathrm{cm}^{3}\right)^{\mathrm{a}}$ & 0.0010 & 0.0008 & 0.0014 \\
$c_{\mathrm{pg}, 0}(\mathrm{~J} / \mathrm{g}-\mathrm{K})^{\mathrm{a}}$ & 1.016 & 1.007 & 0.592 \\
$\left(c_{\mathrm{p}} / c_{\mathrm{v}}\right)_{g, 0}{ }^{\mathrm{a}}$ & 1.40 & 1.58 & 1.58 \\
$\left(\rho_{g} D_{g}\right)_{s, 0}(\mathrm{cP})^{\mathrm{a}}$ & 0.0071 & 0.0070 & 0.0085 \\
$\left(\alpha_{g}\right)_{s, 0}\left(\mathrm{~cm}^{2} / \mathrm{s}\right)^{\mathrm{a}}$ & 0.298 & 0.553 & 0.278 \\
$L e_{s, 0}^{\mathrm{a}}$ & 3.75 & 5.75 & 4.05 \\
\hline
\end{tabular}

a - based on thermophysical models discussed in section 2.0. 


\section{Supplementary Material}

This supplementary material is provided to describe the methodology utilized to analyze data from a rapid compression expansion machine (RCEM), where this integrated analysis could be expanded to account for evaporative cooling during wet compression and then employed for comparisons with chemical kinetic predictions using a zero-dimensional modeling framework. Two example cases are illustrated in Figs. S1 and S2 which demonstrate the methodology. The approach described here has the capability to account for heat release that may occur during the piston compression process, which can be problematic for conventional RCM analyses. The approach is slightly different from conventional methods since it employs well-resolved measurements of both reaction chamber pressure as well as instantaneous piston position. Most RCMs do not have the capability for accurate piston position diagnostics, and because of this the reaction chamber volume histories are often inferred from just the pressure measurement during non-reacting experiments (e.g., see ref. [16]). Knowledge of both is necessary however in order to properly segregate thermo-physical processes such as mass/enthalpy transfer to the piston's crevice/ringpack and evaporative cooling, from the chemical processes leading to low temperature heat release and ignition. Accurate data reduction is necessary for comparisons to chemical kinetic models. In the approach described here physics-based models, in contrast to empirical methods typically utilized for RCMs [16,30], are integrated into a multi-zone analysis of the experiment; these models could be analogously utilized in a single-zone chemical kinetics model. The methodology has been reported previously in refs. [56-58,61], and a review is also presented here.

The primary objective of the data analysis is to determine the time-varying temperature of the reaction chamber gas, as well as (low temperature) heat release or bulk combustion rates so that these can be compared to chemical kinetic predictions. Examples of temperature calculations are illustrated in Figs. S1 and S2. Both mass-averaged (i.e., from P-V data) and adiabatic-core gas temperatures have been utilized for RCM analyses; recent investigations however, have favored the use of adiabatic-core temperatures $[16,30]$. The adiabatic-core approach assumes that the bulk of the reaction chamber gas is insulated from the colder walls of the chamber, and that the effects of heat loss from the non-core, 
boundary layer gas can be modeled as a volumetric, isentropic expansion process for the main chamber gas. Some aspects of this modeling approach are utilized in the analysis presented here where a 'constant-entropy' process is employed.

To compute the gas temperature with this methodology, the reaction chamber is divided into three zones where these include an unreacted zone, a reacted zone, and a mixed zone; the mixed zone is associated with mass that is transferred to the crevice/ringpack regions during compression and ignition, and returns on piston expansion. Multiple zones within the crevice/ringpack volumes can be utilized, as discussed shortly. During piston compression and expansion the cylinder zones are assumed to undergo 'constant-entropy' processes. To account for heat loss a number of approaches could be used including: modifying the zonal specific entropy at each time step, e.g., $\Delta \mathrm{q}_{\text {heat }} / \mathrm{T}_{\text {zone }}$, modifying the compression work term, e.g., $P\left(A_{\text {pist }} V_{\text {pist }}+q_{\text {heat }} V^{\gamma} / \mathrm{P}_{\text {ini }} V_{\text {ini }}{ }^{\gamma}\right)$, or modifying the crevice loss terms to account for the reaction chamber pressure reductions. In typical creviced piston RCMs the crevice losses account for nearly $20 \%$ of the total compression work losses [62].

Within each zone the composition is assumed to be homogenous and no inter-zonal heat transfer is allowed. The partial pressures of the zones in the main charge are summed to give the total cylinder pressure. Mass transfer occurs to the reacted zone due to chemical reaction, and to/from the crevice/ringpack zones due to pressure driven flow. Unreacted, reacted and mixed zone gases are all allowed to enter the crevice, where the proportions from each depends on their respective mass fractions within the cylinder; gas returning from the crevice is sent to the mixed zone. The overall rate of mass transfer to/from the crevice is determined by a crevice/ringpack sub-model. For aerosol operation an additional zone could be coupled with the unreacted zone in order to model the liquid (i.e., droplet) phase where heat and mass transfers could be employed to account for processes that occur during vaporization. The transient wet compression model discussed in this article, or a reduced-order version (e.g., tabulated or even quasi-steady), could be coupled with the zonal calculations to predict the rate of liquid reduction. Heat release, or bulk reaction rates are inferred by matching the summed zonal pressures to the experimentally measured pressure. 
A ring-dynamic sub-model has been developed for the RCEM to account for mass flows into and out of the crevice/ringpack. The sub-model has been described and validated previously [63] and it employs a series of segregated volumes which represent the various 'pockets' within an RCEM's piston ringpack; machined RCM crevices could be modeled using one or two zones. The pressures of the ringpack volumes are dynamic and change based on the time-varying flow rates into and out of the 'pockets'. The 'pockets' or 'gaps' are not in pressure equilibrium with the main reaction chamber due to the lag in pressurization/depressurization, however the gas is assumed to be in thermal equilibrium with the walls of the ringpack due to the very high surface area to volume ratios in these regions. The ring-dynamic submodel also takes into account axial translation of the rings within the grooves of the ringpack and the effects that these movements have on the changing flow paths. Effects of ring heating, due to the unlubricated walls of an RCEM, are also taken into account by modifying the flow rates and the enthalpy of the reemerging crevice charge. Data from non-reactive and reactive charges have been used to calibrate this sub-model.

A sub-model for heat transfer to the reaction chamber walls is also utilized in the data analysis. A detailed study described in ref. [64] calibrated a modified Woschni model [65] to heat flux measurements derived from surface thermocouple data in the RCEM. This correlation can be utilized in a number of ways, as mentioned previously, however better agreement with chemical kinetics predictions is generally observed to be achieved by lumping the effects of heat loss with the crevice/ringpack flows, and utilizing the unmodified, isentropic temperature.

Two representative cases are presented in Figs. S1 and S2 to demonstrate this methodology and illustrate comparisons of RCEM data with associated chemical kinetics predictions. In these figures calculated experimental temperatures are presented as a function of instantaneous effective compression ratio. The effective compression ratio takes into account the varying mass within the main reaction chamber, as in Eq. (3). Mass-averaged temperatures are presented here, and these are nearly identical to the isentropic temperatures; this indicates that heat transfer, especially during compression, is not a significant loss term ( $\sim 3 \%$ of the compression work), while mass transfer to the crevice/ringpack is 
substantial ( $\sim 10-20 \%)$. Results from a 0D, variable volume, homogeneous reactor model (HRM) are also included for comparison. The framework for the HRM is the HCT software [66]. The piston trajectory is integrated into the calculation as a moving boundary for the reactor model. The ring-dynamic sub-model is utilized to modify the piston's experimental velocity history so that the reduction in pressure due to mass transfer to the ringpack is simulated. The crevice transfer process slightly increases the 'dwell' time near maximum compression (since there is significant mass flow at high pressure), and it can quench chemical reaction on expansion since the pressure drops more rapidly than if no crevice flow existed.

In Fig. S1 results for a lean hydrogen + 'air' mixture are illustrated. The ignition / oxidation kinetics for hydrogen have been well studied and a number of detailed, or comprehensive mechanisms are available for comparison / validation [67-70]. Four of these are used here. The piston position histories for the four simulated cases shown here are slightly modified so that similar temperature vs. $C R_{\text {eff }}$ curves are achieved near maximum compression. It can be seen in this figure that the four mechanisms employed yield slightly different behavior where the Saxena et al. mechanism [70] predicts ignition at the lowest temperature while the $\mathrm{Li}$ et al. mechanism [69] requires the most compression heating to achieve ignition. A difference in ignition temperature of about $50 \mathrm{~K}$ is noted between these cases. The RCEM experimental temperature lies within the results of the four mechanisms and this provides a degree of validation for the data analysis methodology.

In Fig. S2 results for the lean premixed $n$-dodecane + 'air' charge presented in Fig. 4 are illustrated. The ignition / oxidation kinetics of $n$-dodecane are not as well studied, though a few kinetic mechanisms have recently been published $[54,71,72]$. Challenges for acquiring requisite validation targets for $n$ dodecane include its significant involatility, as discussed throughout this paper. In the experimental results presented here the LTHR and second stage heat release are identified where these are seen to occur at $T_{\text {LTHR }} \sim 740 \mathrm{~K}$ and $T_{2 n d-s t a g e} \sim 975 \mathrm{~K}$, respectively. A slower reaction process is observed after the second stage. This 'third-stage' of heat release is unusual, however similar behavior has been seen in analogous RCM experiments using $n$-heptane + 'air' mixtures [58], during IC engine operation under some HCCI conditions $[59,60]$, and within flow reactors using DME and $n$-heptane $[73,74]$. The detailed kinetic 
model of Westbrook et al. [54] (2115 species, 8157 reactions) is employed for comparison. The simulation results at these conditions indicate that the LTHR and second stage heat release are predicted to occur at slightly higher temperatures than in the RCEM experiment, at $T_{L T H R} \sim 810 \mathrm{~K}$ and $T_{2 n d-\text { stage }} \sim$ $1060 \mathrm{~K}$, respectively. This is consistent with comparisons to recent $n$-dodecane shock tube data where longer ignition delay times are seen in the model [75]. A 'third-stage' heat release process is not prominent in the kinetic model. Additional experimental data are required in order to understand the discrepancies observed in this dataset. Experiments utilizing the aerosol fueling method described / investigated in this work and others $[14,17,18]$ could be used to acquire such data. The analysis methodology reviewed in this Supplementary Material could be used to account for the effects of vaporization and evaporative cooling during aerosol RCM experiments. 